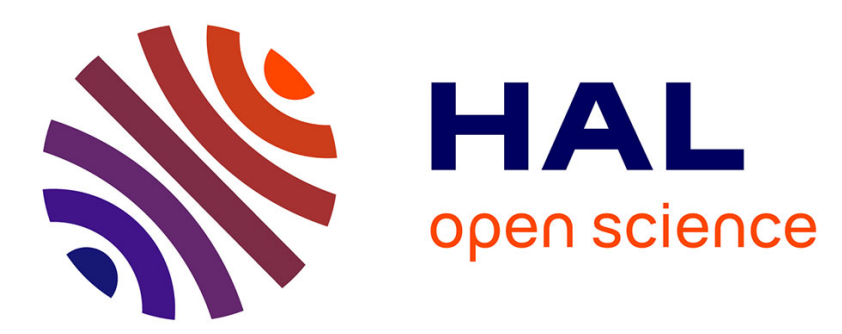

\title{
Robust Hybrid Control of an Atomic Force Microscope for the Characterization of Interaction Force Regions at the Nanoscale
}

Jonathan Cailliez, Mokrane Boudaoud, Shuai Liang, Stéphane Régnier

\section{- To cite this version:}

Jonathan Cailliez, Mokrane Boudaoud, Shuai Liang, Stéphane Régnier. Robust Hybrid Control of an Atomic Force Microscope for the Characterization of Interaction Force Regions at the Nanoscale. IEEE Transactions on Control Systems Technology, 2020, pp.1 - 15. 10.1109/tcst.2020.2985007 . hal-03194770

\author{
HAL Id: hal-03194770 \\ https://hal.science/hal-03194770
}

Submitted on 9 Apr 2021

HAL is a multi-disciplinary open access archive for the deposit and dissemination of scientific research documents, whether they are published or not. The documents may come from teaching and research institutions in France or abroad, or from public or private research centers.
L'archive ouverte pluridisciplinaire HAL, est destinée au dépôt et à la diffusion de documents scientifiques de niveau recherche, publiés ou non, émanant des établissements d'enseignement et de recherche français ou étrangers, des laboratoires publics ou privés. 


\title{
Robust Hybrid Control of an Atomic Force Microscope for the Characterization of Interaction Force Regions at the Nanoscale
}

\author{
Jonathan Cailliez ${ }^{\circledR}$, Mokrane Boudaoud ${ }^{\circledR}$, Shuai Liang, and Stéphane Régnier
}

\begin{abstract}
This article deals with a novel hybrid control of an atomic force microscope (AFM) for the robust characterization of interaction tip/sample force regions. The hybrid structure is composed of several position and force controllers and a specific switch compensation structure. A single selected controller is online at a time, and a robust bumpless switch between different control laws is designed. The objectives are the robustness of each controller and switch compensator with respect to uncertain parameters of the AFM. The method uses discrete points in the range of uncertainties. For each operating point, the hybrid controller is designed by an eigenstructure assignment. The method is generalized by a multimodel approach considering only uncertainties defined by a worst-case analysis, thus reducing the conservatism. The order of the controller is related to the number of observers selected by the user. This offers the possibility to design a low-order controller depending on the control specifications. The experimental results demonstrate the effectiveness of the hybrid controller for a fully automated landing procedure of the AFM tip on a sample surface and for force distance curve cycle characterization. Thanks to the robustness of the control method, landing and cycles are reproducible despite actuator uncertainties, friction variation due to aging, and instrumental issues such as the real-time data acquisition.
\end{abstract}

Index Terms-Atomic force microscope (AFM), hybrid control, nanorobotics, piezoelectric actuator.

\section{INTRODUCTION}

$\mathbf{T}$ HE atomic force microscope (AFM) is a key tool to explore, image, and manipulate a variety of samples at the nanometer scale [1]. Almost all AFMs require accurate feedback control schemes to achieve the high-quality and speed imaging performances [2], [3]. Control methods can be used to either land precisely the AFM cantilever on the sample surface or for the scanning. The latter issue is the key technology for the high-resolution and reliable

Manuscript received May 6, 2019; accepted February 28, 2020. Manuscript received in final form March 29, 2020. This work was supported in part by the research program Investissements d'avenir through the Robotex Equipment of Excellence under Grant ANR-10-EQPX-44 and in part by the research program ANR JCJC Robine under Grant S19JRAR086. Recommended by Associate Editor G. Pin. (Corresponding author: Jonathan Cailliez.)

The authors are with the Institut des Systèmes Intelligents et de Robotique, UMR 7222, Sorbonne Université, F-75005 Paris, France (e-mail: cailliez@isir.upmc.fr; mokrane.boudaoud@isir.upmc.fr; liang@isir.upmc.fr; stephane.regnier@upmc.fr).

Color versions of one or more of the figures in this article are available online at http://ieeexplore.ieee.org.

Digital Object Identifier 10.1109/TCST.2020.2985007 imaging [4], [5]. The former one greatly reduces the AFM tip or sample damage but relies mainly on the operator experience and semiautomated methods [6].

This article will mainly focus on the $\mathrm{Z}$ control of an AFM tip for a safe and fast landing procedure. The literature shows that landing the tip of an AFM or more generally a micrometer-sized probe tool on a sample is often done manually using a haptic interface [7] or a remote control [8]-[10] or using some homemade automated toolboxes but without a specific dynamic contact force control [11]. For automated procedures, a dual stage composed of a coarse actuator and a fine actuator mounted in serial can be used [6], [12], [13]. In [6], the work deals with the definition of the most appropriate signal to use for a reliable contact detection depending on the operating mode of the AFM. For instance, in amplitude modulation AFM, since the phase velocity is proportional to the force gradient near the contact, it provides an accurate information about the contact. An iterative method for a safe landing of the AFM tip is proposed in [13]. First, a fine stage holding the AFM cantilever is driven with a closed-loop force controller. If the fine actuator reaches its maximal extension, the force controller is disconnected, and the coarse actuator is moved forward with an amplitude lower than the maximum extension of the fine actuator. Hence, the tip-sample contact can only happen during the motion of the fine positioner which ensures a secure contact detection. The operation is repeated until the contact is detected. This procedure can take a very long time depending on the initial tip-sample distance and the extension range of the fine actuator. A similar method has been proposed in the previous work [14] using stick-slip type actuators. A faster method is proposed in [15]. The AFM tip is charged to generate the electrostatic forces between the tip and the sample. Such forces can be detected further from the sample than intermolecular forces. The coarse positioner is moved forward until electrostatic forces are detected, and then, the method described in [13] can be used when the tipsample distance is very small which reduces the detection time. In [12], a force controller is used, and its output is filtered by a low-pass filter to drive the coarse actuator and a highpass filter to drive the fine actuator. This method only allows to control the tip-sample force and not the tip position, thus complicating the characterization of interaction force regions. Most of the methods reported in the literature do not discuss 


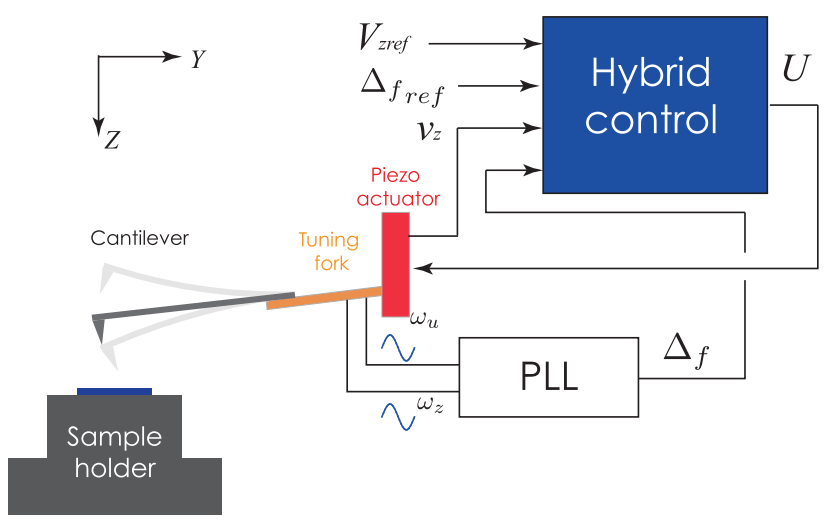

Fig. 1. AFM based on a tuning fork with a hybrid control for closed-loop landing procedure.

the switching dynamic of the AFM actuator when the contact happens and the robustness issues of the closed-loop control.

In this article, AFM tip landing is studied through a new robust hybrid control methodology (Fig. 1). The hybrid controller is composed of multiple controllers and a supervisor with a set of rules to switch between controllers. The issue is that at the time of switching, the controller output is not guaranteed to be smooth, meaning that the derivative of the control signal at the time of switching is very high. This undesired behavior can lead to plant instability, output saturation, or system damage. Many structures have been proposed in the field of hybrid control to ensure bumpless switching between controllers, such as integral structure between the controllers and the plant [16], feedback loop to control the output of the offline controllers [17], multicontroller structure [18], resetting approach of the state of the offline controller at the time of the switch [19], and antiwindup scheme [20]. An integral action after the switch [16], [21] is easy to implement and guarantees zero signal error, but it constraints the controller design. Controller resetting [22] consists in computing a state for the next online controller compatible with the current state of the system and its input. This strategy requires a state-space representation of each controller. Furthermore, it constraints the time between switches to be at least equal to the controlled plant response time. The feedback control is the most flexible technique. The working principle is that a compensator is designed for each controller. The goal is that the output of each offline controller follows the output of the online one. As this strategy reduces the bumpless transfer problem to a simple tracking problem, many strategies can be used such as proportional controller [23], LQ controller [17], and sliding mode controller [24]. The LQ strategy involves a high conservatism and requires an accurate knowledge of the plant. Bumpless transfer through a sliding mode strategy is a robust technique against the parametric variations of the plant, but its main drawback is the shattering. The proportional controller induces a tradeoff between the closed loop bandwidth and the stability margin. Some other methods can also be used to deal with robustness issues [25]. None of these methods have the property of been robust and low conservative.

The proposed methodology of this article aims to design a robust and low conservative hybrid controller. The basis of

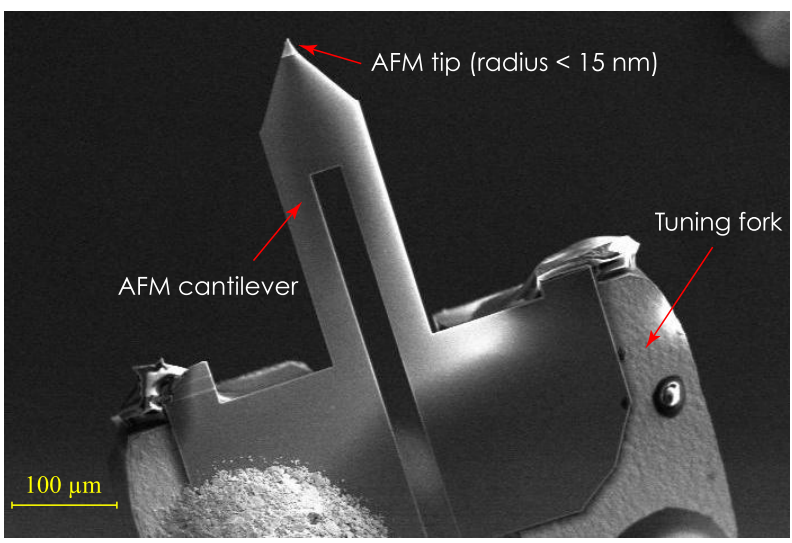

Fig. 2. View of the AFM tuning fork probe inside a scanning electron microscope.

this approach has been reported in [26] and [27] and has been studied in the case of microrobotics in the previous works [28], [29]. This methodology is extended here for hybrid control issues. The hybrid structure is composed of position and force controllers and a specific switch compensator. A robust bumpless switch between different control laws is designed. For the synthesis of each subcontroller, discrete points in the range of the plant uncertainties are used. For each operating point, the hybrid controller is designed by an eigenstructure assignment (ESA) and a multimodel approach to deal with uncertainties defined by a worst-case analysis.

This article is organized as follows. Section II describes the experimental setup and the AFM modeling with uncertainties consideration. The robust hybrid control issue is presented in Section III, and the description of the proposed method is detailed in Sections IV and V. The controller is validated in Section VI considering the specific case of AFM tip landing. The robust characterization of interaction force regions is described in Section VII.

\section{EXPERIMENTAL SETUP AND UNCERTAIN SYSTEM MODELING}

\section{A. Experimental Setup}

The AFM system is composed of three piezoelectric inertia actuators assembled in series to from a XYZ stage and an Akiyama tuning fork probe [30] mounted on the $Z$ actuator. The $\mathrm{Z}$ actuator has a travel range of $12 \mathrm{~mm}$ in the coarse operating mode and a travel range of $\sim 1.5 \mu \mathrm{m}$ in the fine operating mode. It moves the tuning fork probe in the vertical direction. A cantilever with a sharp tip is fixed on the two prongs of the tuning fork as shown in Fig. 2.

The tuning fork probe is made up of two coupled resonators (Fig. 3). The silicon cantilever can be described by the resonator $\left(m_{1}, k_{1}\right.$, and $\left.c_{1}\right)$ and the tuning fork by the resonator $\left(m_{2}, k_{2}\right.$, and $\left.c_{2}\right)$. The typical resonance frequency of the silicon cantilever is $\sim 50 \mathrm{kHz}$ and that of the tuning fork before the cantilever was glued is $\sim 32 \mathrm{kHz}$.

In order to track the resonance frequency of the tuning fork probe, the following steps are followed. First, a frequency analysis without tip/sample contact is performed to get the natural resonance frequency of the tuning fork probe as well 


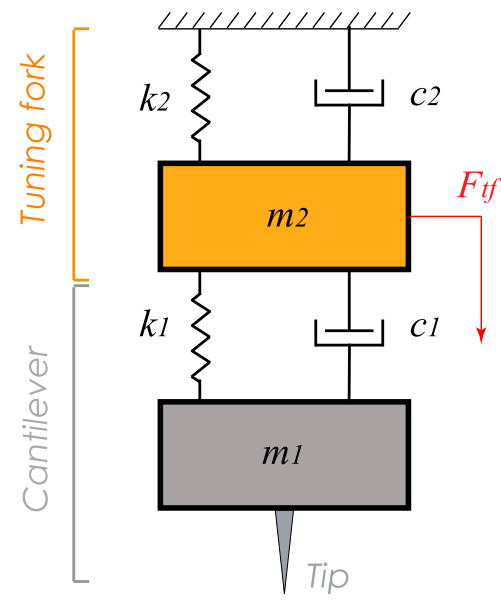

Fig. 3. Simplified model of an Akiyama tuning fork probe. $\mathrm{F}_{\mathrm{tf}}$ is the driving force generated by piezoelectric effect of the tuning fork.

as the associated phase shift. Then, a phase-locked loop (PLL) is programed to keep the phase shift at a constant value and the tuning fork probe oscillations at its resonance frequency. The input voltage of the PLL voltage-controlled oscillator (VCO) is recorded as a measurement of the frequency shift. There is a linear relationship between frequency shift $\Delta f$ and the voltage measurement $V_{\text {sh }}$, i.e., $\Delta f=K_{\mathrm{tf}} V_{\text {sh }}$, where

$$
K_{\mathrm{tf}}=\frac{\Delta f_{\max }}{U_{r}}
$$

where $\Delta f_{\max }$ is the frequency shift measurement span specified by the user and $U_{r}$ is the input voltage span of the $\mathrm{VCO}$, here $10 \mathrm{~V}$. When the tip-sample distance is of few nanometers, the attractive and repulsive interaction forces are detected, which cause a shift of the resonance frequency of the tuning fork probe. For force measurement or for imaging, this resonance frequency is tracked by the PLL. A feedback control of the tip-sample distance is used to keep this resonance frequency at a constant reference value.

In this article, the tip-sample distance is controlled by the piezoelectric inertia $\mathrm{Z}$ actuator whose resonance frequency is around $6 \mathrm{kHz}$ [14]. Initially, the tip-sample distance can be of several millimeters. The tip can be moved toward the sample by means of mixed coarse/fine positioning strategy [14]. In the final fine step positioning (i.e., just before the tip-sample contact), a secure and robust contact detection is required to avoid damaging the AFM tip. Indeed, the radius of the Akiyama cantilever tip is lower than $15 \mathrm{~nm}$. This radius can be highly increased if the cantilever tip abruptly touches the sample. This will cause a loss of the AFM imaging resolution.

\section{B. Uncertain Model $G(\Delta)$}

The dynamic state-space model $G(\Delta)$ of the AFM system is described by the following equation:

$$
G(\Delta):\left\{\begin{array}{l}
\dot{X}=A(\Delta) X+B(\Delta) U \\
v_{z}=C(\Delta) X
\end{array}\right.
$$

where $X \in \mathbf{R}^{n}$ is the state vector, $A \in \mathbf{R}^{n \times n}$ is the state matrix, $B \in \mathbf{R}^{n \times m}$ is the input matrix, and $C \in \mathbf{R}^{p \times n}$ is the output matrix.
TABLE I

PARAMETERS UnCERTAinties OF $G(\Delta)$

\begin{tabular}{llll}
$\begin{array}{l}\text { Uncertain } \\
\text { parameter }\end{array}$ & Description & $\begin{array}{l}\text { Min } \\
\text { value }\end{array}$ & $\begin{array}{l}\text { Max } \\
\text { value }\end{array}$ \\
\hline$\Delta(1)$ & $\begin{array}{l}\text { Fundamental } \\
\text { resonance frequency }\end{array}$ & $\begin{array}{l}2.2 \\
\mathrm{kHz}\end{array}$ & $\begin{array}{l}5.95 \\
\mathrm{kHz}\end{array}$ \\
& Static gain & $\begin{array}{l}12.4 \\
\mathrm{~nm} / \mathrm{V}\end{array}$ & $\begin{array}{l}32.4 \\
\mathrm{~nm} / \mathrm{V}\end{array}$ \\
& & $\begin{array}{l}0.05 \\
\mathrm{~ms}\end{array}$ & $\begin{array}{l}0.25 \\
\mathrm{~ms}\end{array}$
\end{tabular}

The input of the model is the driving voltage $U$ of $\mathrm{Z}$ actuator. The maximum admissible value of $U$ is $100 \mathrm{~V}$. The output $v_{z}$ of $G(\Delta)$ is the displacement in the vertical direction of the $\mathrm{Z}$ actuator, on which the tuning fork probe is fixed. This displacement is measured with an integrated optical encoder sensor of 5-nm resolution.

The dynamic of the tuning fork probe is neglected because its resonance frequency is well above that of the piezoelectric inertia actuator. Therefore, the model $G(\Delta)$ of the AFM describes exclusively the dynamic of the $\mathrm{Z}$ inertia actuator that holds the tuning fork probe.

The uncertainty vector $\Delta$ includes three uncertain parameters $\Delta(1), \Delta(2)$, and $\Delta(3)$ describing, respectively, the uncertain fundamental resonance frequency of $\mathrm{Z}$ actuator, the uncertain static gain of $\mathrm{Z}$ actuator, and the measurement delay of the actuator displacement as shown in Table I. $\Delta(1)$ is included only in the matrix $A, \Delta(2)$ is included only in the matrix $B$, and $\Delta(3)$ is included only in the matrices $A$ and $C$.

1) Uncertainty $\Delta(1)$ : The working principle of piezoelectric inertia actuators is based on a friction force produced by a piezoelectric element to drive a slider. The dynamic of such actuators is related to the parameters of its internal structure (i.e., dimensions, mass, and so on) and may also have a relation with the friction parameters [14]. All these parameters are highly uncertain due to the fabrication process. These actuators are also sensitive to aging due to the friction-based working principle. The first uncertain parameter is the uncertainty of the resonance frequency of $\mathrm{Z}$ actuator. The fundamental resonance frequency of three different actuators of the same reference (SmarAct SLC-1720) has been considered to define the range of variation of $\Delta(1)$ as shown in Table I. The values of the resonance frequencies have been identified in [14].

2) Uncertainty $\Delta(2)$ : Piezoelectric inertia actuators are nonlinear due to a hysteresis. It is the relation between the displacement of the slider and the input voltage of the piezoelectric element. In a previous work [31], the hysteresis of the three actuators has been modeled through a multilinear approximation and identified experimentally. It consists of an approximation of the static hysteresis by several straight lines around operating points. The slope of each straight line can be considered as a static gain of the actuator. The effect of the hysteresis will then be considered in this article as an uncertainty $\Delta(2)$ of the static gain. 


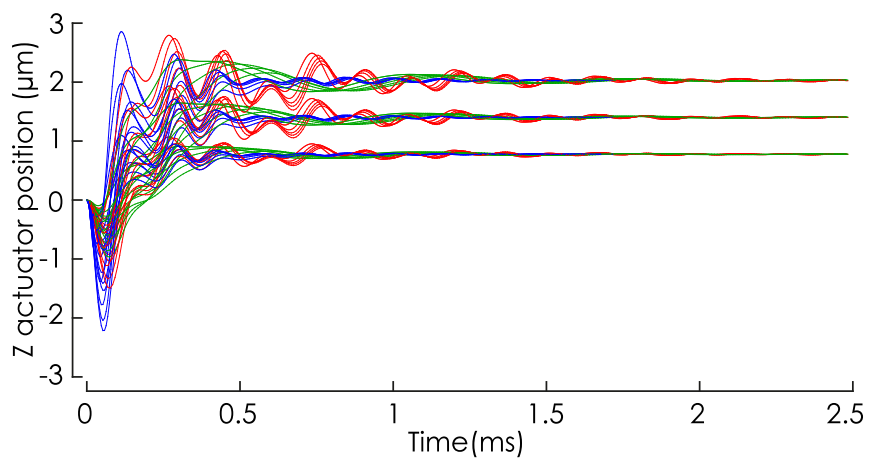

Fig. 4. Step responses of all the considered LTI models.

3) Uncertainty $\Delta(3)$ : The third uncertain parameter $\Delta(3)$ is the measurement delay $T_{r}$ induced by the optical encoder and the measurement chain, i.e., interpolation, counter, and so on. To model this delay, a Padé approximation is added to the model of (2) by means of the following transfer function:

$$
H(s)=\frac{1-\frac{T r}{2} \times s}{1+\frac{T r}{2} \times s}
$$

where $s$ is the Laplace variable.

The actuator dynamics and the static hysteresis have been modeled and identified in a previous work [31]. The results are shown in Table I.

\section{Definition of a Finite Number of LTI Models From $G(\Delta)$}

Instead of considering all the values of each uncertainty in the range of variation (see Table I), the discrete points in the range of uncertainties are defined to build a set of linear time-invariant (LTI) models from $G(\Delta)$.

The uncertainty of the resonance frequency is taken into account by considering the dynamics of the three actuators SLC-1720. The fundamental resonance frequency of the actuators $1-3$ are, respectively, $2.2,1.5$, and $5.95 \mathrm{kHz}$.

For each actuator dynamics, and therefore, for each resonance frequency, six different values of the static gain equally separated in the range $[12.4-32.4 \mathrm{~nm} / \mathrm{V}]$ are considered.

For each actuator dynamics and each value of the static gain, five values of the measurement delay equally separated in the range $(0.05-0.25 \mathrm{~ms})$ are considered.

Finally, taking into account the combination of the dynamics (i.e., resonance frequency) of each of the three actuators, the six different values of the static gain and the five different values of the measurement delay, $3 \times 6 \times 5=90$ LTI models, are defined to form a set of models describing $G(\Delta)$.

Each LTI model has seven states, i.e., six states to describes three eigenmodes and one state for the Padé approximation. The step responses of all the 90 open-loop models are shown in Fig. 4.

\section{HYBRID CONTROL ISSUE}

The methodology for a safe contact detection in the fine positioning mode of the AFM tip is proposed through the robust hybrid control. The inputs of the hybrid control (Fig. 1)

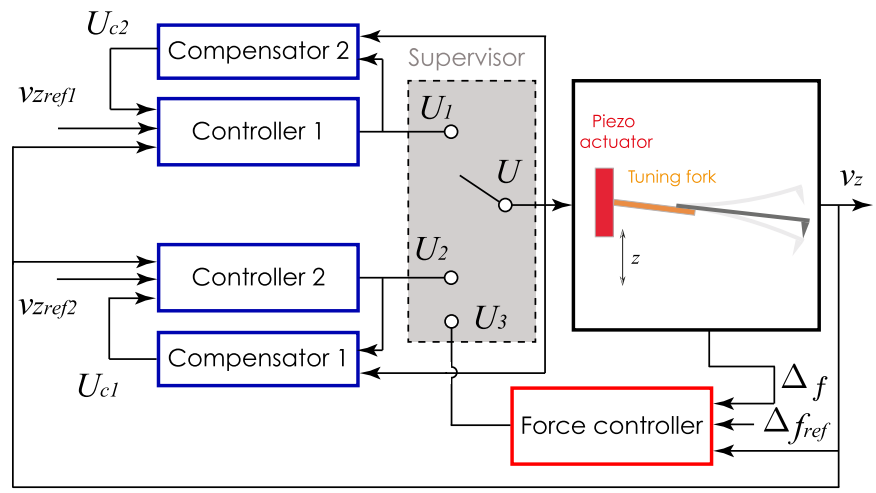

Fig. 5. Hybrid control scheme of the AFM treated here. The design of the position controllers 1 and 2 is presented in Section VI-A. The design of the force controller is presented in Section VII-B. The detailed internal structure of the force controller is shown in Fig. 20(a). An SEM image of the AFM tuning fork is presented in Fig. 2.

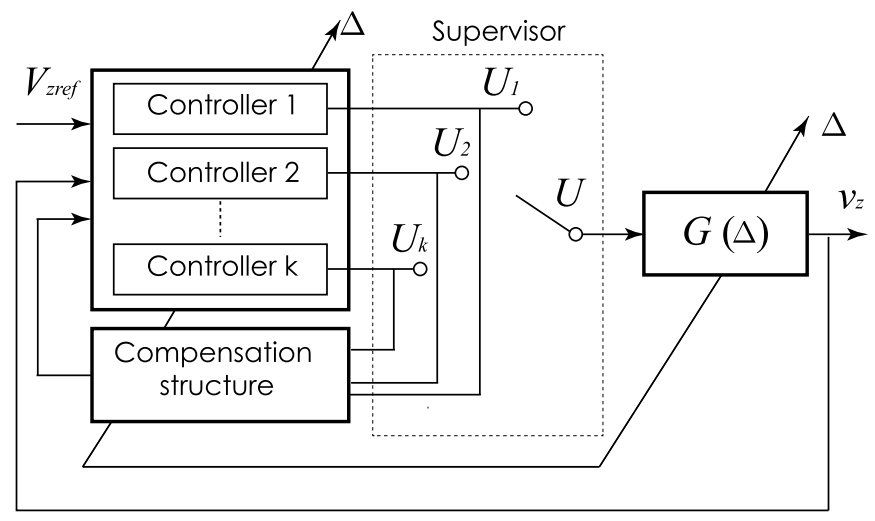

Fig. 6. General hybrid control scheme of an uncertain system $G(\Delta)$. The more specific control scheme treated in this article including position and force control is shown in Fig. 5.

are the position reference of the $\mathrm{Z}$ actuator $V_{z_{\text {ref }}}$ and its realtime position (i.e., actual position) $v_{z}$, the desired shift of the resonance frequency of the tuning fork $\Delta_{\text {fref }}$, and its real-time value $\Delta_{f}$. Its output is the control voltage $U$ that feeds the $\mathrm{Z}$ actuator.

The hybrid control structure includes in addition to the position controllers, a force controller that will be described in Sections VI-A and VII-B, respectively. The control diagram is shown in Fig. 5, where $V_{z_{\text {ref }}}=\left[\begin{array}{ll}v_{z_{\text {ref } 1}} & v_{z_{\text {ref } 2}}\end{array}\right]^{T}$.

Let us consider the control structure of Fig. 6, where an uncertain system $G(\Delta)$ is controlled by a set of $k$ controllers providing control signals $U_{1}, U_{2}, \ldots$, and $U_{k}$. At any time, only one controller is activated by a supervisor, it is said to be online, and the others are said to be offline. The aim is that all the closed-loop systems of the hybrid structure satisfy the robustness specifications with respect to (w.r.t) uncertain parameters of $\Delta$. The switch between the different control signals must be achieved at any time with the small possible discontinuity of $U$.

The switch compensation structure must be also robust. It must be designed to track the output of the online controller with the output of the offline ones.

Control Method: Each controller and compensator of the hybrid control scheme of Fig. 6 can be designed by many 
robust control methods to deal with the system uncertainties. Classical robust controllers such as those based on the $H_{\infty}$ with LMI constraints take into account a continuum of values in the range of variation of $\Delta$ and are highly conservative. The multimodel approach proposed in this article uses a different approach by considering only a finite number of selected uncertainties to obtain a robust controller. This is a progressive method that is first designed on a nominal model and then adds robustness step by step based on a worst case analysis. It does not require the treatment of a continuum of models in the range of variation of $\Delta$ to satisfy the robust performances with respect to the uncertainties but just a wellselected number of relevant worst case models. This approach has many advantages that will be discussed in Section VIII.

This multimodel method has been first developed to deal with control issues in the field of aeronautics [26], [27]. It has then been applied with an experimental validation in the field of microsystems, and especially, for those involving nonlinear mechanical suspensions [28], [29]. This method has, nevertheless, not been designed to deal with hybrid control issues. Therefore, the first contribution of this article is the generalization of the method in the case of hybrid control as described in Section V. This generalization has been first presented in the conference [32], and it is generalized here, where switch conditions are not only defined by position measurement of a piezoelectric inertia actuator but also by data from a tuning fork-probe of the AFM. The application of this method for robust AFM tip landing and the characterization of interaction force regions are the second contribution of this article. It is the first time that such a low conservative control method is applied in the field of AFM whose advantages are discussed in Section VIII.

The hybrid control structure includes in addition to the position controllers, a force controller with a feedback signal from the tuning fork-based sensor as shown in Fig. 5. This generalization is of importance for a secure and robust landing of the AFM tip. A precise characterization of attractive and repulsive tip-sample interaction forces regions is thereafter proposed and validated experimentally using the proposed control method.

\section{Robust Control By ESA And Multimodel FORMULATION}

The aim of this section is to define the basis of the multimodel control with an ESA and an observer-based structure.

Let us consider the dynamic model of the AFM $G(\Delta)$ with uncertain parameters included in a vector $\Delta$ as described in (2). The objective is to assign a set of eigenstructures (i.e., eigenvalues and eigenvectors) for the closed-loop system. The variations of $\Delta$ must be taken into account during the control design to satisfy the robustness specifications.

In the sequel, the control design will be described for a frozen value of $\Delta$, and then, the method will be generalized considering the variation of the uncertain parameters.

\section{A. Closed-Loop Control for a Frozen Value of $\Delta=\Delta_{l}$}

The classical ESA methodology is based on the following Lemma [33]. Let us consider the triplet $\left(\lambda_{i}, v_{i}, \omega_{i}\right)$ with $\lambda_{i}$ an eigenvalue, $v_{i}$ an eigenvector, and $\omega_{i}$ an input direction, satisfying

$$
\left[A\left(\Delta_{1}\right)-\lambda_{i} I \quad-B\left(\Delta_{1}\right)\right]\left[\begin{array}{c}
v_{i} \\
\omega_{i}
\end{array}\right]=0 .
$$

This triplet is assigned in closed loop by the constant gain $K$ if and only if

$$
K C\left(\Delta_{1}\right) \nu_{i}=\omega_{i} .
$$

With the classical ESA, it is not possible to assign more eigenstructures than the number of measurable outputs of the plant (2), and this is the degree of freedom constraint [26]. Moreover, the assignment does not guarantee the closed-loop robustness against the variations of $\Delta$ [28], [29].

The robustness issue can be solved by a multimodel design as explained in Section IV-B. To deal with the degree of freedom, the dedicated observers can be designed. One possible solution for the observation is to estimate a linear combination of the states $X$ through the following model:

$$
\left\{\begin{array}{l}
\frac{d z_{i}}{d t}=\pi_{i} z_{i}-t_{\pi_{i}} v_{z}+u_{\pi_{i}} B\left(\Delta_{1}\right) U \\
z_{i}=u_{\pi_{i}} X
\end{array}\right.
$$

where $z_{i}$ is the output of the observer.

This model is based on a modal approach for observer design [34]. It shows that a linear combination of the states $u_{\pi_{i}} X$ can be estimated by an observer increasing the number of outputs. It allows assigning as many more eigenstructures as the number of observations.

The observer is obtained through the vector $u_{\pi_{i}} \in \mathbf{C}^{n}$, the vector $t_{\pi_{i}} \in \mathbf{C}^{p}$, and a complex number $\pi_{i} \in \mathbf{C}$ satisfying

$$
u_{\pi_{i}} A\left(\Delta_{1}\right)+t_{\pi_{i}} C\left(\Delta_{1}\right)=\pi_{i} u_{\pi_{i}} .
$$

where $n=6$ and $p=1 . \pi_{i}$ is the pole of the observer that defines its dynamics.

The pole $\pi_{i}$ must be different from the poles of $G\left(\Delta_{1}\right)$.

Let $q$ be the number of eigenstructures that have to be assigned and $p$ be the number of nonredundant outputs of the plant (2). If $q>p, n_{0}=q-p$ observers have to be designed.

The following notations will be used to define a set of $n_{0}$ observers:

$$
\begin{gathered}
U_{o}=\left[\begin{array}{c}
u_{\pi_{1}} \\
\vdots \\
u_{\pi_{n_{o}}}
\end{array}\right] \quad T_{o}=\left[\begin{array}{c}
t_{\pi_{1}} \\
\vdots \\
t_{\pi_{n_{o}}}
\end{array}\right] \quad \Pi_{o}=\left[\begin{array}{ccc}
\pi_{1} & \ldots & 0 \\
\vdots & \ddots & \vdots \\
0 & \ldots & \pi_{n_{o}}
\end{array}\right] \\
U_{o} \in \mathbb{C}^{n_{o} \times n}, \quad T_{o} \in \mathbb{C}^{n_{o} \times p}, \text { and } \Pi_{o} \in \mathbb{C}^{n_{o} \times n_{o}} .
\end{gathered}
$$

Now, it is possible to define an augmented system $G\left(\Delta_{1}\right)$ with a set of $n_{o}$ observers to have additional outputs $Z$ with

$$
Z=\left[\begin{array}{c}
z_{1} \\
z_{2} \\
\vdots \\
z_{n_{o}}
\end{array}\right] .
$$


Using (2) and (6) with the notation of (8) and (9), the statespace representation of the augmented model is given as follows:

$$
\left\{\begin{array}{l}
{\left[\begin{array}{c}
\dot{X} \\
\dot{Z}
\end{array}\right]=\left[\begin{array}{cc}
A\left(\Delta_{1}\right) & 0_{n \times n_{o}} \\
-T_{o} C\left(\Delta_{1}\right) & \Pi_{o}
\end{array}\right]\left[\begin{array}{l}
X \\
Z
\end{array}\right]+\left[\begin{array}{c}
B\left(\Delta_{1}\right) \\
U_{o} B\left(\Delta_{1}\right)
\end{array}\right] U} \\
{\left[\begin{array}{c}
v_{z} \\
Z
\end{array}\right]=\left[\begin{array}{c}
C\left(\Delta_{1}\right) \\
U_{o}
\end{array}\right] X .}
\end{array}\right.
$$

Thanks to the separation principle, the control problem consists, now, of finding the static gain $K$ to assign the eigenstructures to the system of equations

$$
\left\{\begin{array}{l}
\dot{X}=A\left(\Delta_{1}\right) X+B\left(\Delta_{1}\right) U \\
v_{z}=C\left(\Delta_{1}\right) X \\
Z=U_{o} X
\end{array}\right.
$$

To deal with precision requirements, i.e., zero closed-loop static error, an integral action can be added. In this case, the number of the states of the model (11) is increased by one.

The control law becomes

$$
U=K_{i} \int\left(v_{z_{\text {ref }}}-v_{z}\right) d t-K_{y} v_{z}-K_{z} Z
$$

where $v_{z_{\text {ref }}}$ is the closed-loop input reference, and the parameters $K_{i}, K_{y}$, and $K_{z}$ are the gains of the controller such as $K=\left[\begin{array}{lll}K_{z} & K_{y} & K_{i}\end{array}\right]$.

It is not needed to add an observer to the system to deal with the integral action.

\section{B. Robust Control by a Multimodel Design}

The discrete values of $\Delta$ in the set $\left[\Delta_{\min }, \Delta_{\max }\right]$ are considered. This allows the definition of $g$ LTI models $G\left(\Delta_{1}\right)$, $G\left(\Delta_{2}\right)$, and, $\ldots, G\left(\Delta_{g}\right)$ from (2). If needed, the number of values of $\Delta$ can be increased around particular points.

In this case, $r_{i}$ ESAs are necessary for each model $G\left(\Delta_{i}\right)$. Therefore, if $g$ LTI models are treated, $\sum_{i=1}^{g} r_{i}$ constraints induced by these assignments must satisfy the following set of equations [see (5)]:

$$
\begin{aligned}
& K C_{\text {int }}\left(\Delta_{1}\right) v_{1, \Delta_{1}}=\omega_{1, \Delta_{1}} \\
& K C_{\text {int }}\left(\Delta_{1}\right) v_{r_{1}, \Delta_{1}}=\omega_{r_{1}, \Delta_{1}} \\
& K C_{\text {int }}\left(\Delta_{g}\right) v_{1, \Delta_{g}}=\omega_{1, \Delta_{g}} \\
& K C_{\text {int }}\left(\Delta_{g}\right) \nu_{r_{g}, \Delta_{g}}=\omega_{r_{g}, \Delta_{g}} .
\end{aligned}
$$

$C_{\text {int }}$ defines the output matrix of the state-space model (2) with an integrator.

To design a set of observers for a model $G\left(\Delta_{i}\right)$, (6) and (7) can be used taking into account the uncertain vector $\Delta_{i}$. Moreover, by refereeing to [34], each assignment is related to a vector $\gamma_{j, \Delta_{i}}$, where

$$
\begin{aligned}
\gamma_{j, \Delta_{i}}=\left(\lambda_{j, \Delta_{i}} I_{n_{o} \times n_{o}}-\Pi_{o}\right)^{-1} & \\
& \times\left(U_{o} B\left(\Delta_{i}\right) \omega_{j, \Delta_{i}}-T_{o} C_{\mathrm{int}}\left(\Delta_{i}\right) v_{j, \Delta_{i}}\right)
\end{aligned}
$$

$I_{n_{o} \times n_{o}}$ is the identity matrix. For each $\Delta_{i}, 1<j<r_{i}$.

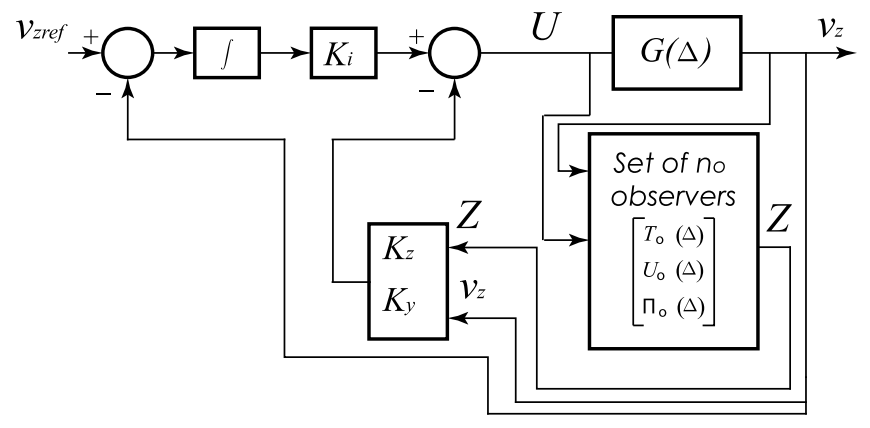

Fig. 7. Robust output feedback control scheme of the uncertain system $G(\Delta)$ with a set of $n_{O}$ observers and an integrator.

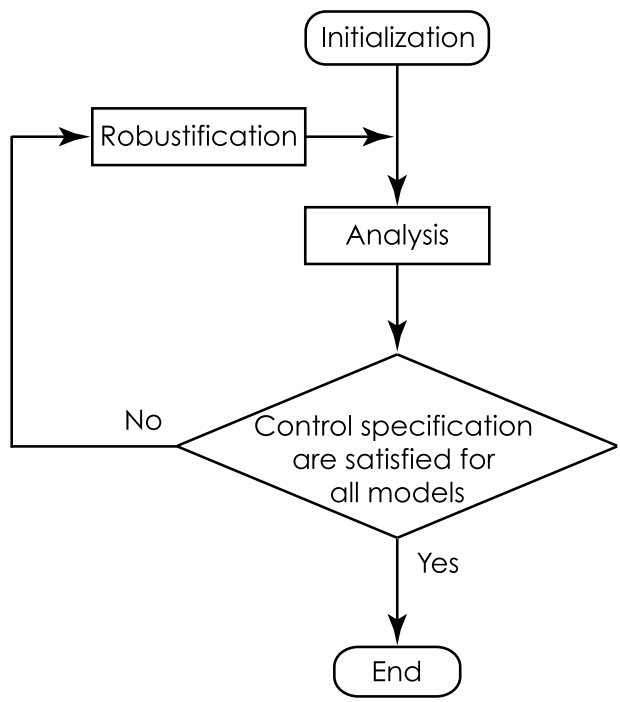

Fig. 8. Flowchart of the robust control synthesis.

$\gamma_{j, \Delta_{i}}$ is fundamental for the computation of the gain $K$.

The closed-loop system fits the constraints (13) if and only if [34]

$K=\left[\begin{array}{c}\omega_{1, \Delta_{1}} \\ \vdots \\ \omega_{r_{g}, \Delta_{g}}\end{array}\right]^{T}\left[\begin{array}{ccc}\gamma_{1, \Delta_{1}} & \cdots & \gamma_{r_{g}, \Delta_{g}} \\ C_{\mathrm{int}}\left(\Delta_{1}\right) v_{1, \Delta_{1}} & \cdots & C_{\mathrm{int}}\left(\Delta_{g}\right) v_{r_{g}, \Delta_{g}}\end{array}\right]^{-1}$

where $K=\left[\begin{array}{lll}K_{y} & K_{z} & K_{i}\end{array}\right]$.

If the models $G\left(\Delta_{i}\right)$ that have been used for the control design are worst case models, the computed gain $K$ can lead to a robust controller with respect to $\Delta$. The block diagram of the output feedback controller is shown in Fig. 7.

The robust control design can be performed iteratively following three main steps [26]: initialization, analysis, and robustification (Fig. 8).

1) Initialization: Definition of $g$ LTI models $G\left(\Delta_{i}\right)$. The selection of any model $G\left(\Delta_{i}\right)$ in the defined set and application of the assignment constraints of (13) in accordance with control specifications. If needed, a set of observers can be designed. Computation of the gain $K$ using (14) and (15).

2) Analysis: Simulation of the pole map of the $g$ closedloop models with the observers and the computed gain $K$. If every model fits the control specifications, then 
finish. Else define a worst case model $G\left(\Delta_{i+1}\right)$ and go to the robustification step. The worst case model is the one whose one of the performances is the farthest from the specification. In the sequel, the worst case model will be the one whose closed-loop pole has the highest real part value.

3) Robustification: Definition of new assignment constraints for the current worst case model and addition of these new constraints to the previous ones to increase the number of equations in (13). If needed, new observers can be computed for the current value of $\Delta$. Computation of a new gain $K$ using (14) and (15) considering the assignment constraints of all the treated models. If needed, quasi-similar assignments can be eliminated. Those assignments tend to lead to unrealistic gains. Then go to the analysis step.

\section{NEW FORMULATION FOR ROBUST HYBRID CONTROL}

The goal is to define a hybrid structure so that each subcontroller and each compensator can be designed by a robust ESA. This way, each controller and compensator as well as the switch between different control laws will be robust against the variations of $\Delta$, and its synthesis will have a low conservatism.

The compensator is a controller that controls the output of the offline controller to follow the output of the online one. As such at the switching time, a bumpless transition of the plant's control signal is satisfied. For instance, in Fig. 9, the compensator $C_{p 1}$ is active when the controller 1 is online. It must ensure a bumpless transfer from $U_{1}$ to $U_{2}$ at the time of the switch. Therefore, it is used to keep $U_{2}$ stable when $U_{1}$ is controlling the plant so that when switching between $U_{1}$ and $U_{2}$ happens, there is as little discontinuity of the control signal driving the plant as possible.

Let us consider the structure of Fig. 6, providing that the controllers are independent. Each controller can be assigned with an independent compensator. Each controller can be designed independently with different control specifications. Each compensator controls the output signal of its associated offline controller to track the output of the online controller. In any case, only one controller is active.

Without the loss of generality, the structure of Fig. 6 will be treated for $k=2$. The hybrid scheme will be composed of two dynamic controllers, namely, controllers 1 and 2 and two compensators $C_{p 1}$ and $C_{p 2}$ as shown in Fig. 9. The supervisor is a high-level control that is based on predefined conditions, i.e., the conditions for which the control law $U$ switches from $U_{1}$ to $U_{2}$ and vice versa.

The internal structure of the controllers 1 and 2 is the same as in Fig. 7 except that the control input is not $v_{z_{\text {ref }}}$, but the signals $v_{z_{\text {ref } 1}}+U_{c 2}$ for the controller 1 and $v_{z_{\text {ref } 2}}+U_{c 1}$ for controller 2.

The observers used to design the controller 1 will be designated as "set of observers 1" with $U_{o_{1}} \in \mathbb{C}^{n_{o_{1}} \times n}, T_{o_{1}} \in$ $\mathbb{C}^{n_{o_{1}} \times p}$, and $\Pi_{o_{1}} \in \mathbb{C}^{n_{o_{1}} \times n_{o_{1}}}$. Those of controller 2 will be designated as "set of observers 2" with $U_{o_{2}} \in \mathbb{C}^{n_{o_{2}} \times n}$,
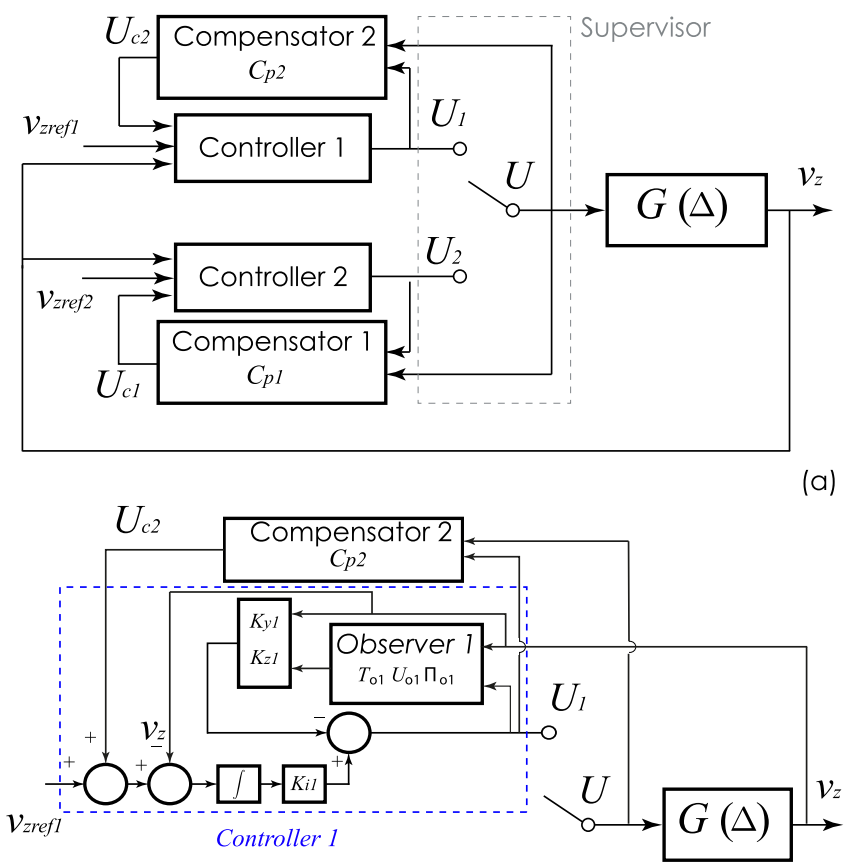

(b)

Fig. 9. (a) Hybrid control scheme of an uncertain system $G(\Delta)$. Each controller and compensator are designed by an ESA and a multimodel formulation. (b) Control part including controller 1 and compensator 2 with a detailed view of the internal structure of controller 1 .

$T_{O_{2}} \in \mathbb{C}^{n_{o_{2}} \times p}$, and $\Pi_{o_{2}} \in \mathbb{C}^{n_{o_{2}} \times n_{o_{2}}}$. The static control gain of controller 1 and controller 2 will be expressed, respectively, as $K_{1}=\left[\begin{array}{lll}K_{z_{1}} & K_{y_{1}} & K_{i_{1}}\end{array}\right]$ and $K_{2}=\left[\begin{array}{lll}K_{z_{2}} & K_{y_{2}} & K_{i_{2}}\end{array}\right]$. In the sequel, the proposed structure of the compensators will be explained considering $C_{p 1}$. The same principle is used to compute $C_{p 2}$.

The compensator $C_{p 1}$ is active when the controller 1 is online. It must ensure a bumpless transfer from $U_{1}$ to $U_{2}$ at the time of the switch. As shown in Fig. 9, the inputs of $C_{p 1}$ are the signals $U$ and $U_{2}$, and its output is the signal $U_{c 1}$. The latter is used to control the dynamic of $U_{2}$ so that it tracks the active signal $U_{1}$. The output $v_{z}$ and the input reference $v_{z_{\text {ref } 2}}$ are considered in the proposed approach as disturbances. Then, the tracking issue can be solved by a robust ESA, providing that a state-space representation of the dynamic system of input $U_{c 1}$ and output $U_{2}$ is defined.

Let us consider the following state vector:

$$
X_{c 2}=\left[\begin{array}{c}
Z_{2} \\
v_{z_{\text {int }}}
\end{array}\right] .
$$

With $Z_{2}$, the output vector of the set of observers 2 and $v_{z_{2_{\text {int }}}}=\int\left(v_{\text {ref }_{2}}-v_{z}+U_{c 1}\right) d t$.

Let us now take into account the $g$ models of $G(\Delta)$ used for the synthesis of controller 2. For each model, $r_{i}$ ESAs have been defined. In this case, $n_{0_{2}}=\left(\sum_{i=1}^{g} r_{i}\right)-p$, where $n_{0_{2}}$ is the number of observers in the set of observers 2 , and $p$ is the number of nonredundant outputs of the plant $G(\Delta)$.

For a model of uncertainty $\Delta_{i}$, the output of the observer is $Z_{2_{i}}$, and its parameters are $\left(U_{o_{2_{i}}}, T_{O_{2_{i}}}\right.$, and $\left.\Pi_{o_{2_{i}}}\right)$. Therefore, the output and the parameters of the set of observers 2 can be 
defined as follows:

$$
\begin{aligned}
& Z_{2}=\left[\begin{array}{llll}
Z_{2_{1}} & Z_{2_{2}} & \cdots & Z_{2_{g}}
\end{array}\right] \\
& U_{o_{2}}=\left[\begin{array}{llll}
U_{o_{2}}^{T} & U_{o_{2}}^{T} & \cdots & U_{o_{2} g}^{T}
\end{array}\right]^{T} \\
& T_{o_{2}}=\left[\begin{array}{llll}
T_{o_{2}}^{T} & T_{o_{2}}^{T} & \cdots & T_{o_{2 g}}^{T}
\end{array}\right]^{T} \\
& \Pi_{o_{2}}=\operatorname{diag}\left(\Pi_{o_{2_{1}}} \Pi_{o_{2_{2}}} \cdots \Pi_{o_{2 g}}\right) \text {. }
\end{aligned}
$$

Using (6), for each value of $\Delta_{i}$, the derivative of $Z_{2_{i}}$ is

$$
\dot{Z_{2_{i}}}=U_{o_{2_{i}}} B\left(\Delta_{i}\right) U_{2}-T_{o_{2_{i}}} v_{z}+\Pi_{o_{2_{i}}} Z_{2_{i}} .
$$

$U_{2}$ can be expressed as

$$
U_{2}=\left[\begin{array}{ll}
K_{z_{2}} & K_{i_{2}}
\end{array}\right] X_{c 2}+K_{y_{2}} v_{z} .
$$

The derivative of $v_{z_{2 \text { int }}}$ can be calculated trivially

$$
v_{z_{\text {int }}}=v_{z_{\text {ref } 2}}-v_{z}+U_{c 1} \text {. }
$$

By substituting the result of (19) in (18), one gets

$$
\begin{aligned}
\dot{Z_{2_{i}}}=U_{o_{2_{i}}} B\left(\Delta_{i}\right) & {\left[K_{z_{2}} K_{i_{2}}\right] X_{c 2} } \\
& +\left(U_{o_{2_{i}}} B\left(\Delta_{i}\right) K_{y_{2}}-T_{o_{2_{i}}}\right) v_{z}+\Pi_{o_{2_{i}}} Z_{2_{i}} .
\end{aligned}
$$

Now, it is possible to combine the results of (20) and (21). One is able to get the derivative of $X_{c 2}$, thus getting the desired state-space representation

$$
\left\{\begin{array}{l}
\dot{X_{c 2}}=A_{c 2} X_{c 2}+B_{c 2} U_{c 1}+B_{c 2}^{\prime} v_{z}+B_{c 2}^{\prime \prime} v_{z_{\text {ref }_{2}}} \\
U_{2}=C_{c 2} X_{c 2}+K_{y_{2}} v_{z}
\end{array}\right.
$$

with

$$
\begin{aligned}
& A_{c 2}=\left[\begin{array}{cc}
U_{o_{2}} B\left(\Delta_{1}\right) K_{z_{2}} & U_{o_{2}} B\left(\Delta_{1}\right) K_{i_{2}} \\
U_{o_{2}} B\left(\Delta_{2}\right) K_{z_{2}} & U_{o_{2}} B\left(\Delta_{2}\right) K_{i_{2}} \\
\vdots & \vdots \\
U_{o_{2 g}} B\left(\Delta_{g}\right) K_{z_{2}} & U_{o_{2 g}} B\left(\Delta_{g}\right) K_{i_{2}} \\
0_{1 \times n_{o_{2}}} & 0_{1 \times 1}
\end{array}\right]+M_{\Pi_{o}} \\
& M_{\Pi_{o}}=\left[\begin{array}{cc}
\Pi_{o_{2}} & 0_{n_{o_{2} \times 1}} \\
0_{1 \times n_{o_{2}}} & 0_{1 \times 1}
\end{array}\right] \\
& B_{c 2}=\left[\begin{array}{c}
0_{n_{o_{2}} \times 1} \\
1
\end{array}\right], \quad B_{c 2}^{\prime}=\left[\begin{array}{c}
U_{o_{2}} B\left(\Delta_{1}\right) K_{y_{2}}-T_{o_{2_{1}}} \\
\vdots \\
U_{o_{2 g}} B\left(\Delta_{g}\right) K_{y_{2}}-T_{o_{2 g}} \\
-1
\end{array}\right] \\
& B_{c 2}^{\prime \prime}=\left[\begin{array}{c}
0 \\
\vdots \\
0 \\
1
\end{array}\right] \\
& C_{c 2}=\left[\begin{array}{ll}
K_{z_{2}} & K_{i_{2}}
\end{array}\right] \text {. }
\end{aligned}
$$

Using this representation, it is possible to seize the problem caused by this type of controller for switching. $A_{c 2}$ has an eigenvalue equal to zero due to the integrator, so if the references for both controllers 1 and 2, i.e., $v_{z_{\text {ref } 1}}$ and $v_{z_{\text {ref } 2}}$ are not the same, $U_{2}$ will diverge if the compensator $C_{p 1}$ is not added to the control scheme. This remark is also valid for $U_{1}$ and the compensator $C_{p 2}$.
Let us recall that, here, $v_{z}$ and $v_{z_{\text {ref } 2}}$ are considered as disturbances in the model. As such, using the separation principle, they are considered for the compensator design as equal to zero. The state-space representation of the tracking problem becomes

$$
\left\{\begin{array}{l}
\dot{X_{c 2}}=A_{c 2} X_{c 2}+B_{c 2} U_{c 1} \\
U_{2}=\left[\begin{array}{ll}
K_{z 2} & K_{i_{2}}
\end{array}\right] X_{c 2}
\end{array}\right.
$$

This model contains $n_{0_{2}}+1$ states.

\section{Robust Hybrid CONTROL FOR AFM TIP LANDING}

The goal of this section is to design the hybrid control method including controller 1 , controller 2, compensator 1 , and compensator 2 of Fig. 5. The force control part will be treated in Section VII.

The controllers 1 and 2 will be designed with the procedure described in Section IV-B based on the set of LTI models derived from Section II-B. The uncertainty $\Delta$ includes the three uncertainties of Table I. Let us recall that $\Delta(1)$ is included only in the matrix $A, \Delta(2)$ is included only in the matrix $B$, and $\Delta(3)$ is included only in the matrices $A$ and $C$ of the state-space model (2) of the AFM.

Each compensator will be designed with an ESA using the model of (23).

Two protocols I and II will be defined to set the switching rules of the supervisor of Fig. 5.

\section{A. Hybrid Control Design}

Based on the LTI models $G\left(\Delta_{i}\right) 1<i<90$, the controller of Fig. 5 will be designed. The controllers 1 and 2 and the force controller will have different closed-loop specifications.

The position control specifications are listed as follows:

1) The controller 1 must satisfy in closed loop no static error, an overshoot less than 5\%, and a settling time lower than $150 \mathrm{~ms}$.

2) The controller 2 must satisfy in closed loop no static error, an overshoot less than $5 \%$, and a settling time lower than $6 \mathrm{~ms}$, i.e., 25 times faster and the closedloop system with the controller 1 .

Indeed, as shown in Fig. 16, the controller 1 will drive the system when the AFM tip is not in the region of interaction forces. A slow response time is preferable to avoid approaching the force regions too fast. However, controller 2 will drive the system when the AFM tip is within the region of interaction forces. A higher closed-loop bandwidth is needed for AFM interaction force characterization.

1) Design of Controller 1: The controller is designed using the methodology of Section IV-B. The nominal model $G\left(\Delta_{1}\right)$ is chosen to be that of the actuator $3\left(\Delta_{1}(1)=5.95 \mathrm{kHz}\right)$ with a static gain equal to $\Delta_{1}(2)=12.4 \mathrm{~nm} /$ Volts and a sensor delay of $\Delta_{1}(3)=0.25 \mathrm{~ms}$. For this nominal model, four eigenstructures are assigned in closed loop with the poles $-20,-300,-2.2 \times 10^{2}$, and $-5 \times 10^{3}$. The model $G\left(\Delta_{1}\right)$ has one measurable output only. Therefore, two observers are designed. 


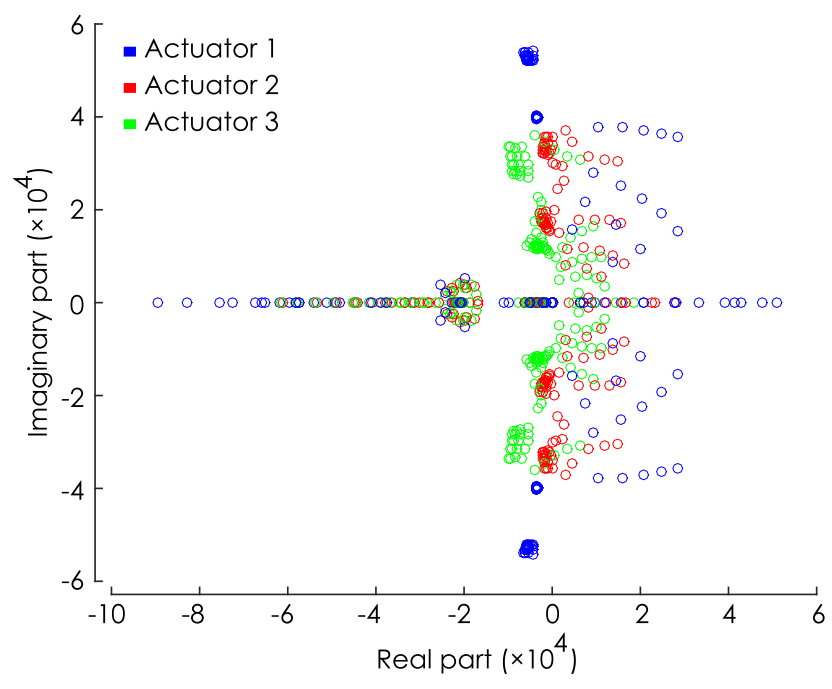

Fig. 10. Pole map of all the closed-loop models with controller 1 at the first synthesis.

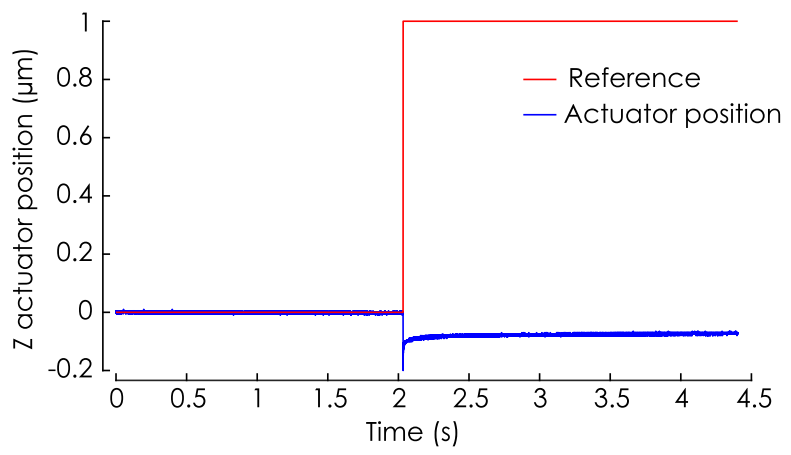

Fig. 11. Step response of one actuator with controller 1 at the first synthesis (experimental result).

Using (15), the gain $K_{1}$ is computed taking into account four assignment constraints. The worst case analysis is performed for the 90 LTI closed-loop models with the computed gain. The pole map of the closed-loop models is shown in Fig. 10. Almost, all the closed-loop models are unstable except for three of them, one is the closed-loop nominal model (i.e., that used for the first synthesis) and the two others do not fit the specifications. An experimental test has been performed with the controller, and the results are shown in Fig. 11. As soon as the reference goes to $1 \mu \mathrm{m}$, the controller diverges. This leads to a saturation of the amplifier, whose output changes abruptly from 0 to $100 \mathrm{~V}$. A slip of the actuator slider occurs.

This result shows that a robustification step is necessary. The worst case model is chosen to be the model of actuator $3\left(\Delta_{1}(1)=5.95 \mathrm{kHz}\right)$ with a static gain equal to $\Delta_{1}(2)=32.4 \mathrm{~nm} / \mathrm{V}$ and a sensor delay of $\Delta_{1}(3)=0.25 \mathrm{~ms}$. For this model, two assignments are added with the poles at -30 and $-5.2 \times 10^{2}$. The new gain $K_{1}$ is computed again using (15). Now, six assignment constraints are considered, and they include the four assignments of the previous nominal model and the two assignments of the worst case model. The step responses of all the closed-loop models with the new computed gain (i.e., after the robustification) are shown

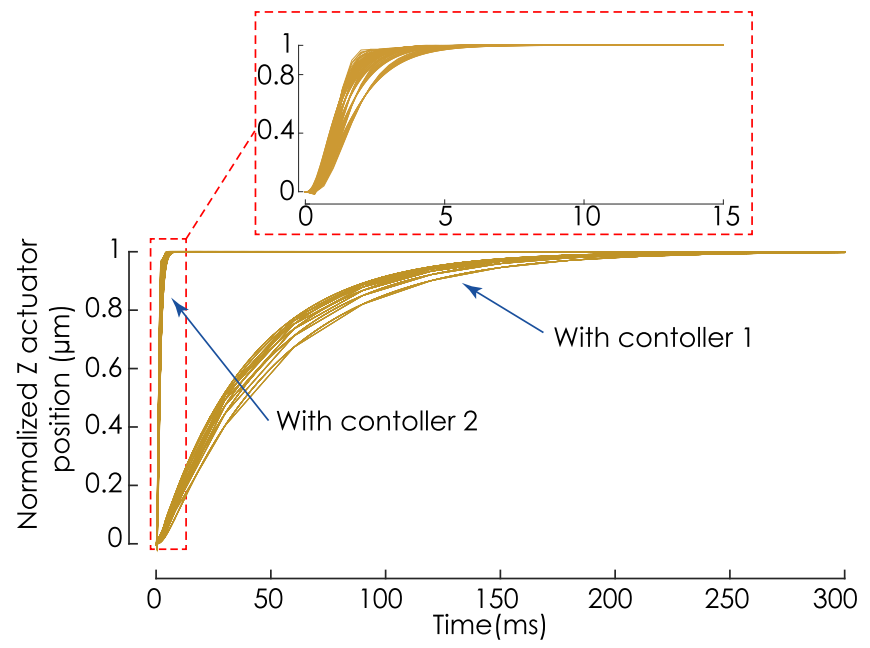

Fig. 12. Normalized to unity step responses of all closed-loop models with controllers 1 and 2 at the second synthesis.

in Fig. 12. All the closed-loop models fit the specifications. The procedure is stopped, and the values for both the static gain and the observer's parameters of controller 1 are kept. This controller is robust with respect to the variations of $\Delta$.

2) Design of Controller 2: The procedure is the same as for controller 1 except for the specifications. Two iterations have been also necessary to satisfy the control specifications in a robust way. The step responses of the closed-loop LTI models with the robust controller 2 are also shown in Fig. 12.

3) Design of the Compensators $C_{p 1}$ and $C_{p 2}$ and Simulation of the Hybrid Position Controller: The model of (23) is used for the design of the compensator. For each compensator, two ESAs are performed with the poles $-5 \times 10^{4}$ and $-4 \times 10^{4}$. The robustness against the variations of $\Delta$ is not needed here as none of the state-space matrices of the model (23) are dependent on the variation of $\Delta$. They are frozen matrices. However, if the controllers 1 and 2 were system dependent, uncertain, or time varying, the robustness would have been possible with the proposed methodology.

The robust hybrid controller including the controllers 1 and 2 and the compensators $C_{p 1}$ and $C_{p 2}$ has been first tested by simulations for each of the 90 LTI models $G\left(\Delta_{i}\right)$. For each model, the supervisor is defined with the following protocol.

Protocol I:

1) Controller 1 is first online with a step input reference of $1 \mu \mathrm{m}$.

2) Once the $Z$ actuator reaches $0.8 \mu \mathrm{m}$, the controller 2 comes online with a step input reference of $0.7 \mu \mathrm{m}$ plus a sine with increasing amplitude.

3) Once the actuator reaches $0.85 \mu \mathrm{m}$, the controller 1 is back online with a step input reference of $0 \mu \mathrm{m}$.

This protocol is of importance for the robust AFM tip landing and for the characterization of attractive and repulsive tip-sample forces as explained in detail in Section VI-B2.

The simulation results of the closed-loop step responses of the 90 LTI models are shown in Fig. 13. The Z actuator follows well the Protocol I in a robust way. The closedloop specifications of the controllers 1 and 2 are satisfied. 


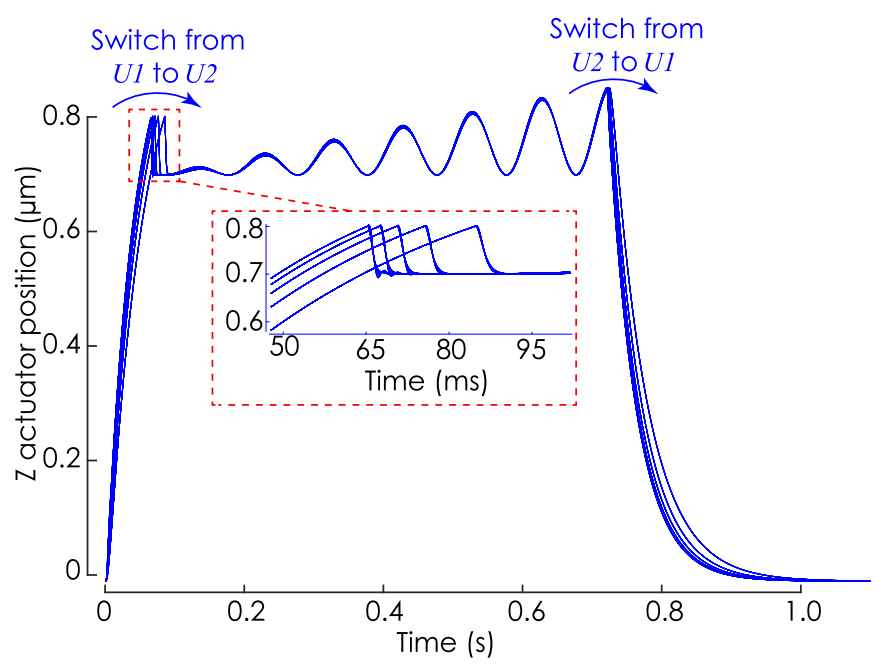

Fig. 13. Closed-loop displacements of all LTI models with the hybrid controller.

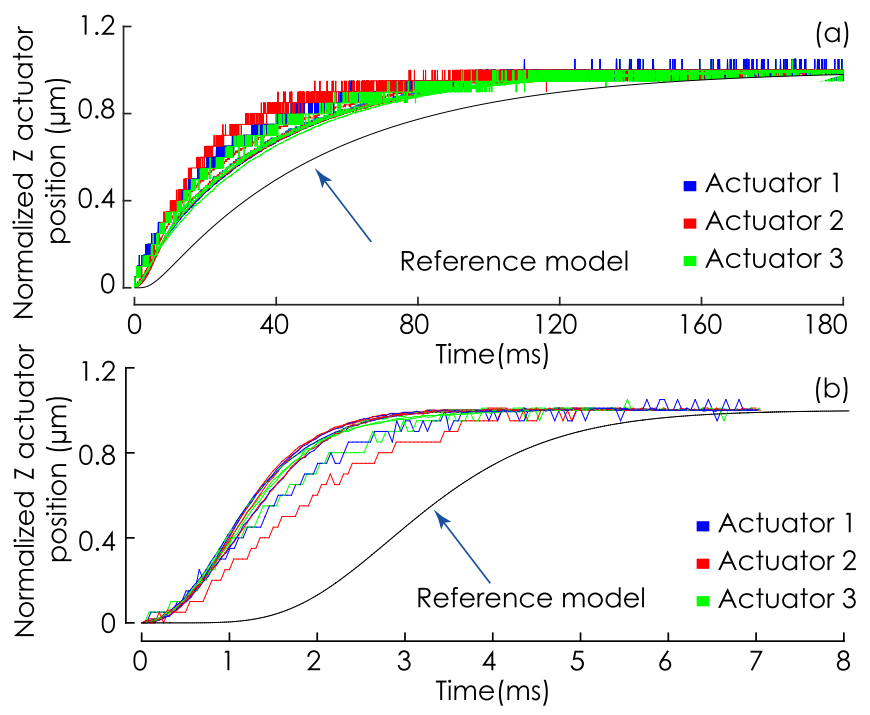

Fig. 14. Normalized to unity step responses of the three actuators controlled with (a) controller 1 and (b) controller 2 at the second synthesis. The step input references are $100 \mathrm{~nm}, 500 \mathrm{~nm}, 1 \mu \mathrm{m}$, and $1.5 \mu \mathrm{m}$. The step response of the reference closed-loop model is shown.

The switch from the controller 1 to controller 2 and vice versa is achieved with no overshoot or no vibrations. The vibrations observed around $0.07 \mathrm{~s}$ are due to the design constraints of controller 2, and their amplitude is below $5 \mathrm{~nm}$.

\section{B. Experiments}

1) Experimental Implementation of the Hybrid Controller and Analysis: The experimental setup includes a processor board with analog-to-digital converters (ADCs) and digital-toanalog converters (DACs), a counter, and an interpolator for the optical encoders of the piezoelectric actuators, a real-time interface, and the three piezoelectric inertia actuators of the same reference (SmarAct SLC-1720). The hybrid controller has been designed using MATLAB/Simulink software and has been implemented, using the Simulink model, to the processor board.

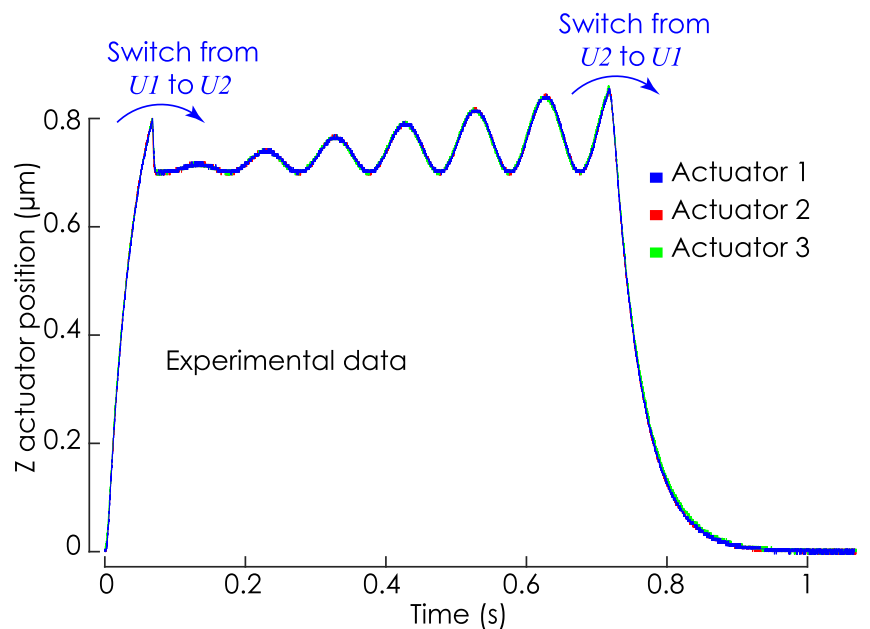

Fig. 15. Experimental closed-loop displacements of the three actuators with the robust hybrid control.

First, the controllers 1 and 2 have been experimentally tested on the three actuators to validate their robustness with respect to the dynamic variations of the friction and the resonance frequency. Different input references have been applied, namely $100 \mathrm{~nm}, 500 \mathrm{~nm}, 1 \mu \mathrm{m}$, and $1.5 \mu \mathrm{m}$. This is done to validate the robustness of the controllers with respect to the hysteresis. The measurement delay is inherent to the acquisition chain, i.e., interpolation, counter, and so on. The experimental closed-loop responses normalized to unity (i.e., divided by the input reference) using the controllers 1 and 2 are shown in Fig. 14(a) and (b), respectively. The curves with noise are those for a step reference of $100 \mathrm{~nm}$. These results validate experimentally the robustness of the controllers 1 and 2 .

In the third experiment, the protocol I has been followed. The experimental results are shown in Fig. 15. They show a bumpless switch before the steady state of the closed-loop systems with the controller 1 (i.e., switch from $U_{1}$ to $U_{2}$ ) and a bumpless switch from $U_{2}$ to $U_{1}$.

The results of Figs. 13 and 15 show that the hybrid control method can be efficiently applied for a large batch of AFM systems despite: 1) parameters variations due to the fabrication process; 2) friction parameters changing due to aging; 3 ) hysteresis; and 4) measurement delay.

2) Protocol for Robust AFM Tip Landing: Let us consider Fig. 16 to illustrate the protocol of robust AFM tip landing. This figure is that of Fig. 15 with an inversion of the coordinate axis " $\mathrm{Z}$ actuator position $(\mu \mathrm{m})$ " to highlight the AFM tipsample distance with an origin to zero distance. The protocol for robust AFM tip landing is, therefore, defined as follows.

\section{Protocol II:}

1) The controller 1 is first online with a step input reference of $1 \mu \mathrm{m}$.

2) When the tuning fork probe detects an attractive force, the controller 2 is switched $\mathrm{ON}$ with a reference equal to the position of the actuator at the switch time minus $0.1 \mu \mathrm{m}$ plus a sine with an increasing amplitude. An attractive force is a force whose effect is to pull the AFM cantilever toward the sample, inducing a negative frequency shift of the tuning fork. Such forces can 


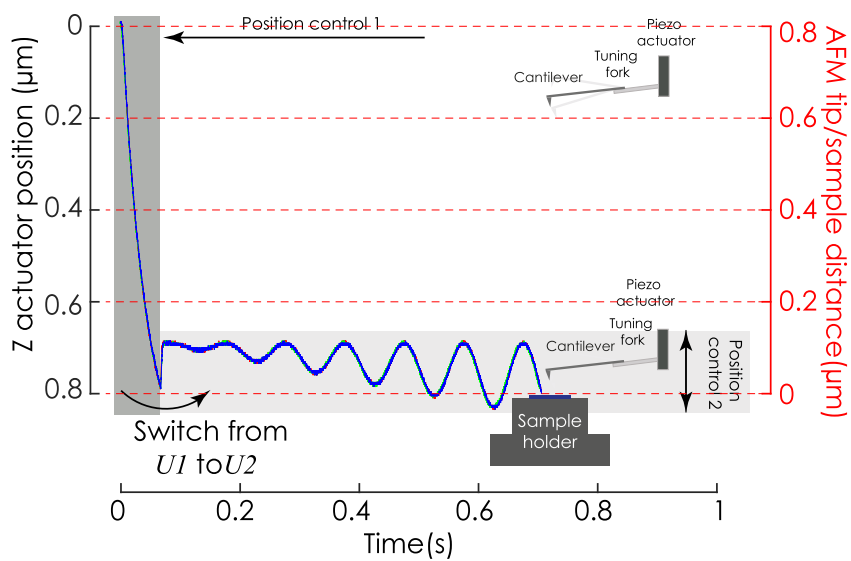

Fig. 16. Experimental closed-loop displacements of the three actuators and protocol for robust AFM tip landing.

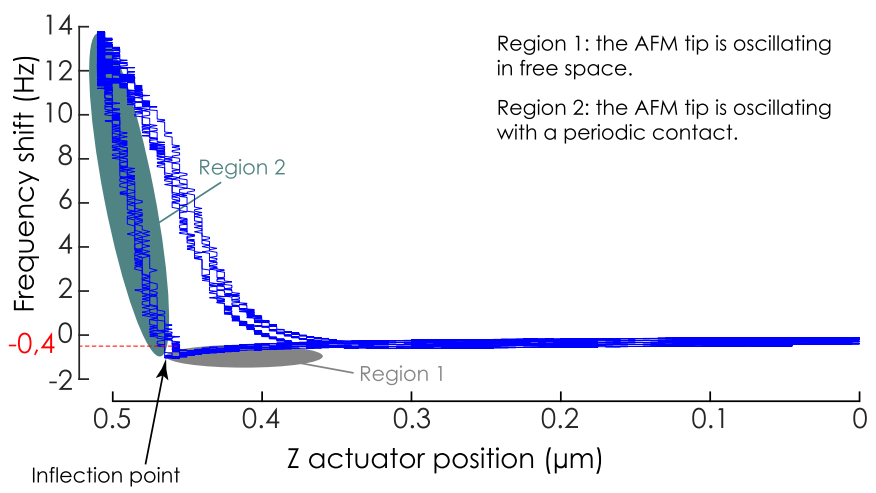

Fig. 17. Tuning fork frequency shift against $\mathrm{Z}$ actuator position for a sine position reference with an amplitude of $500 \mathrm{~nm}$ and an angular frequency of $1 \mathrm{rad} / \mathrm{s}$. The inflection point defines the limits of regions 1 and 2 .

be electrostatic or van der Waals forces for instance. The supervisor informs the hybrid controller about the contact when a shift of the resonance frequency $\Delta_{f}$ of the tuning fork is measured. The sine with an increasing amplitude reference allows a characterization of attractive and repulsive force regions in a smooth way.

3) When $\Delta_{f}$ reaches a threshold value corresponding to a repulsive force due to a real tip-sample contact, the controller 1 is back online with a step input reference of $0 \mu \mathrm{m}$. As shown in Fig. 17, the limit between the free space region and the periodic contact region is at the inflection point that occurs at $-0.8 \mathrm{~Hz}$. The condition considered here is for the threshold value $\Delta_{f}=-0.4 \mathrm{~Hz}$.

This protocol will be expanded with more constraints in Section VII to consider also the force control.

The efficiency of the switch from the controller 1 to controller 2 when the condition is provided by the tuning fork has been tested experimentally. The results are shown in Fig. 18. The dashed curves are those of the controlled three actuator positions when the switch condition is that when the amplitude displacement is equal to $0.8 \mu \mathrm{m}$. The continuous curve is that of the displacement of the $\mathrm{Z}$ actuator within the AFM when the switch condition is provided by $\Delta_{f}$. For visual comparison

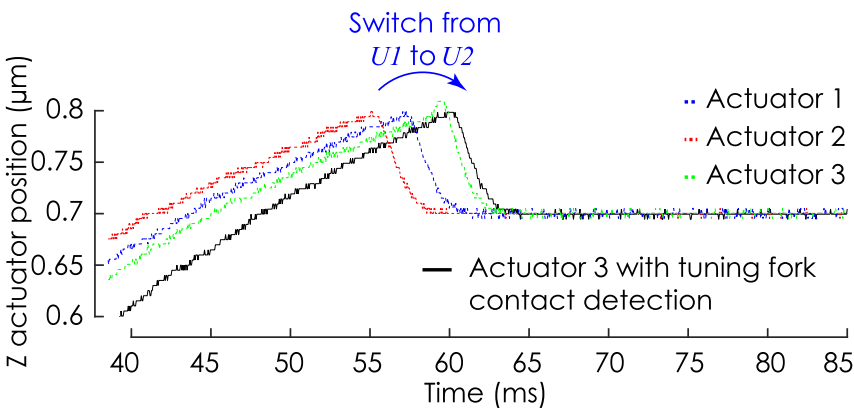

Fig. 18. Experimental closed-loop displacements of the three actuators at the time of the switch from the controller 1 to controller 2 . The continuous curve is that of the displacement of the $\mathrm{Z}$ actuator within the AFM when the switch condition is provided by the contact detection, thanks to the measurement of $\Delta_{f}$.

reasons, the continuous curve is shifted in $X$ - and $Y$-axes so that the maximum of the curve is equal to $0.8 \mu \mathrm{m}$ and the switch time is close to that of the other curves. The experimental results show that even in the presence of forces exerted on the AFM tip, the switch is achieved in a bumpless way.

\section{ROBUST CHARACTERIZATION OF INTERACTION ForCE REgIONS AND CONSTANT FREQUENCY-SHIFT CONTROL}

\section{A. Force Regions Characterization With the Controller 2}

The characterization starts when the AFM tip is within the region of interaction forces. For that purpose, the first steps of protocol II are applied, i.e., the controller 1 is first online with a step input reference of $1 \mu \mathrm{m}$, and when the tuning fork-probe detects an attractive force, controller 2 is switched ON with a reference equal to the position of the actuator at the switch time minus $0.1 \mu \mathrm{m}$. The state of the system at that moment is the initial conditions for the force characterization.

Now, only the controller 2 is active. A sinusoidal input position reference with a fixed frequency and amplitude is applied. The AFM tip is in a noncontact mode, and it is oscillating around its initial state. During the motion of the $\mathrm{Z}$ actuator, the frequency shift of the tuning fork is measured. The contact is considered when the frequency shift is at $-0.4 \mathrm{~Hz}$. The frequency shift/Z actuator position is extracted experimentally for two different angular frequencies of the sinusoidal input reference, namely, 0.5 and $5 \mathrm{rad} / \mathrm{s}$, and for three different amplitudes, namely, $1 \mu \mathrm{m}, 200 \mathrm{~nm}$, and $150 \mathrm{~nm}$.

Before obtaining exploitable results, the slow drift of the frequency shift has been managed by a posttreatment of the signal. This treatment consists of a statistical analysis of the signal and a removal of the linear tendency of the time series. The results after the treatment for the two angular frequencies are shown in Fig. 19(a) and (b). The approach-retract characteristic exhibits a hysteresis for an angular frequency of $5 \mathrm{rad} / \mathrm{s}$, while no hysteresis is observed for $0.5 \mathrm{rad} / \mathrm{s}$.

\section{B. Force Control}

Sections VI and VII-A have shown that the position of $\mathrm{Z}$ actuator holding the tuning fork-probe can be controlled efficiently by the robust hybrid controller, and the interaction 

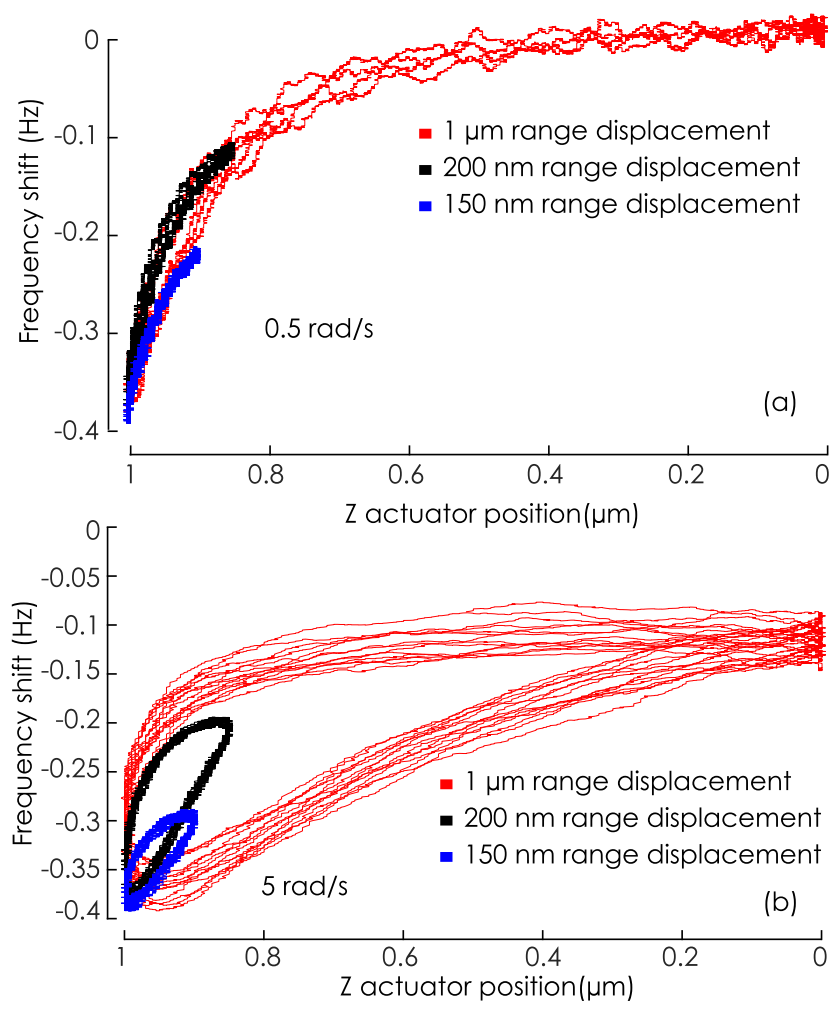

Fig. 19. Tuning fork frequency shift against $\mathrm{Z}$ actuator position for sine wave amplitudes of $1 \mu \mathrm{m}$ (red), $200 \mathrm{~nm}$ (dark), and $150 \mathrm{~nm}$ (blue). The results are shown for sinusoidal angular frequencies of (a) 0.5 and (b) $5 \mathrm{rad} / \mathrm{s}$. For visual comparison reasons, the curves related to 200- and 150-nm range displacements have been shifted in $y$-axis so that their $x$-coordinates for $-0.4 \mathrm{~Hz}$ frequency shift are the same as for the curves related to $1-\mu \mathrm{m}$ range displacement.

forces near the contact and in contact can be efficiently characterized in a robust way despite the AFM parametric uncertainties. The goal, now, is to complete the protocol II with the aim of a frequency modulation AFM by the force controller of Fig. 5. The detailed internal structure of the force controller is shown in Fig. 20(a). In its initial state, the AFM tip is within the region of interaction forces.

The frequency modulation AFM consists of keeping the frequency shift at a desired value with a feedback control on the $\mathrm{Z}$ actuator. To do so, the human operator can decide to switch from $U_{2}$ to $U_{3}$ by acting on the selector of Fig. 20(a). When the force controller is offline and the controller 2 is online, the selector is in position 2 , and the memory function is a unit gain. When the force controller is online, the selector is in position 1, and the memory function keeps the value of

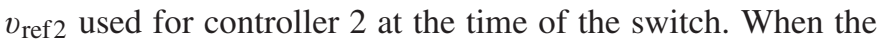
force controller is online, its inputs are the measured frequency shift $\Delta_{f}$, the frequency shift reference $\Delta f_{\text {ref }}$, and the measured $\mathrm{Z}$ actuator position $v_{z}$. Its output is the control voltage $U_{3}$.

The internal structure of the force controller is composed of the controller 2 and a PID controller. The strategy consists of tracking the desired shift of frequency of the tuning fork probe $\Delta f_{\text {ref }}$ with the PID controller to define the reference position for the $\mathrm{Z}$ actuator. The latter is the output of the PID controller. Then, the robust controller 2 will drive the $\mathrm{Z}$ actuator to reach this reference position which corresponds

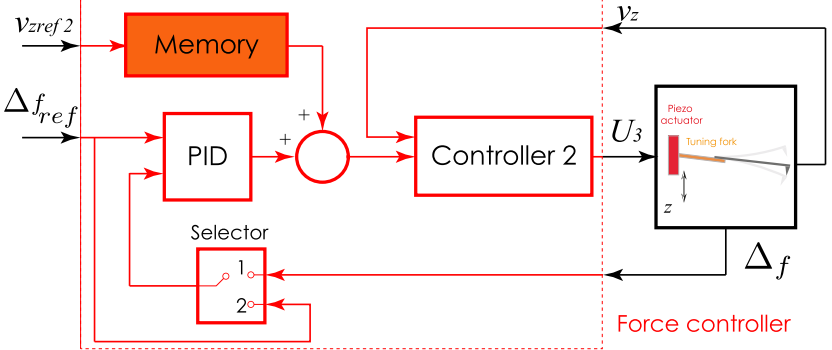

(a)

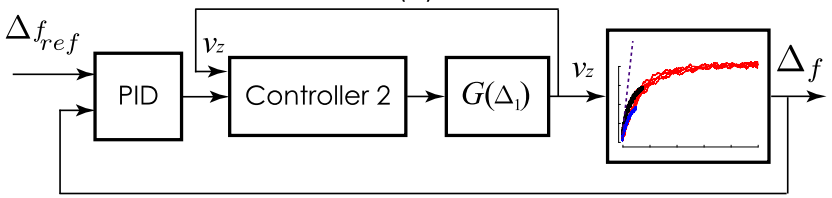

(b)

Fig. 20. (a) Proposed structure for frequency modulation AFM with the force controller. When the force controller is online, the selector is in position 1 and the memory function keeps the value of $v_{\text {ref }}$ used for controller 2 at the time of the switch. (b) Closed-loop system used to tune the parameters of the PID controller by simulation.

to the desired $\Delta f_{\text {ref }}$. In this way, the issues related to AFM uncertainties and measurement delays are avoided by the same way as before, thanks to the robustness of the controller 2 .

To define the parameters of the PID controller, the specifications are a closed-loop response time higher than $0.1 \mathrm{~s}$ and a phase margin of $90^{\circ}$. The specification of the response time is done to avoid being affected by the hysteresis as shown in Fig. 19(b).

The function PID tuner of MATLAB [35] has been used to find the parameters of the PID controller, which meet the specifications. To this end, the model of Fig. 20(b) has been built, where $G\left(\Delta_{1}\right)$ is the nominal model used in Section VI-A1. To define the relation between $\Delta_{f}$ and $v_{z}$, the results of Fig. 19(a) are used. The curve of Fig. 19(a) is divided into several straight lines around operating points. The slope of each straight line can be considered as a static gain. The highest static gain is considered for the synthesis of the PID controller [Fig. 20(b)].

The bumpless transfer between the controller 2 and the force controller is very critical here because the AFM tip is very close to the sample. It is satisfied by choosing the error input of the PID controller to be 0 when it is not active. When switched $\mathrm{ON}$, the previous reference for the controller 2 is stored and added to the controller output as shown in Fig. 20(a). For the experimental validation, a scan of the attractive force region is first made using the controller 2 with a sinusoidal input reference as in Section VII-A. Then, at an arbitrary time, the user can decide to switch to a force control to keep the frequency shift at the current value, i.e., the value of $\Delta_{f}$ at the time of switching. The results are shown in Fig. 21.

Thanks to these results, one can define a complete new protocol for robust AFM tip landing and force characterization as follows.

\section{Protocol III:}

1) Detection of the Interaction Force Region: Initially, the AFM tip can be several hundred micrometers away 


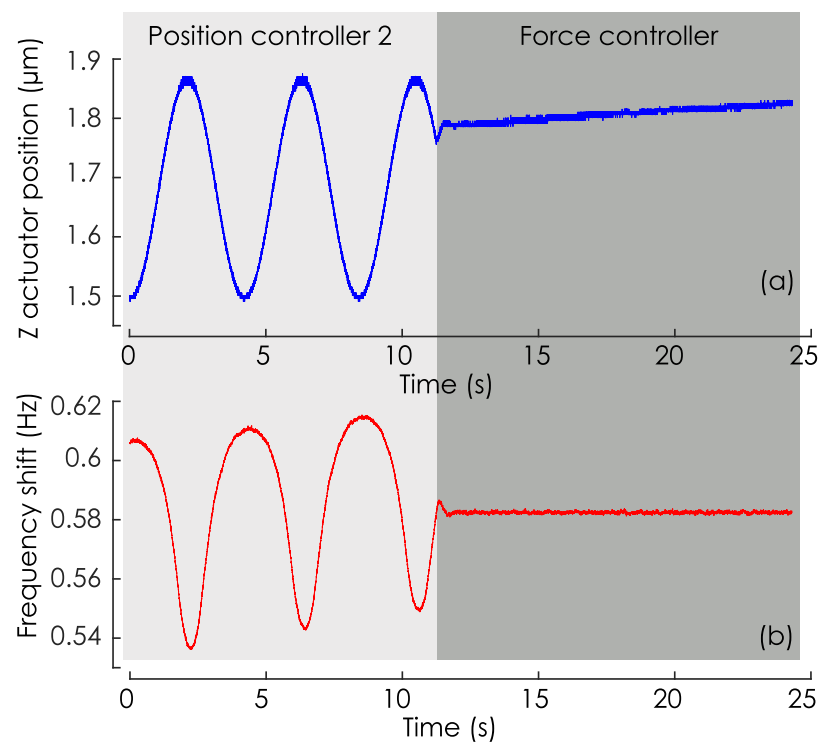

Fig. 21. (a) Experimental $\mathrm{Z}$ actuator position and (b) experimental frequency shift of the tuning fork probe during the position/force control.

from the sample. The tip can be moved toward the sample by means of mixed coarse/fine positioning strategy with the $\mathrm{Z}$ inertia actuator [14] as explained in Section I. The controller 1 is applied for each fine positioning. In the final fine step positioning, (i.e., just before the tip-sample contact), the hybrid control scheme of Fig. 5 can be used.

2) Characterization of the Tuning Fork Probe Frequency Shift Against Z Actuator Position: when the tuning fork probe detects an attractive force, the controller 2 is switched on with a reference equal to the switch position minus $0.1 \mu \mathrm{m}$ plus a sine with an increasing amplitude and an angular frequency around $0.5 \mathrm{rad} / \mathrm{s}$ to avoid the hysteresis of Fig. 19(b). In this way, the relation $\Delta_{f} / v_{z}$ as in Fig. 19(a) can be defined.

3) Force Control for Frequency Modulation AFM: The PID substructure of the force controller can be tuned online taking into account the highest slope of the relation $\Delta_{f} / v_{z}$. Then, the user can decide to switch from $U_{2}$ to $U_{3}$ by acting on the selector of Fig. 20(a). A frequency modulation AFM can then be started.

\section{DISCUSSION}

The control method presented in this article has advantages and drawbacks. The main drawback is that it relies on the experience of the designer on the system to be controlled, especially for the definition of the set of $g$ LTI models to be treated and the selection of the worst case models which are not known a priori and are defined step by step. The number $g$ of LTI models will define the resolution of the multimodel synthesis. The more the value of $g$ is high, the highest will be the models considered for the worst case analysis. It is mainly related to the number of discrete values selected between the lower and the upper bounds of each uncertain parameter.

The proposed control method has, however, several advantages that are as follows.
1) The method only considers closed-loop specifications and design constraints imposed by the user, thus leading to a higher potential of specifications in closed loop with a controller order that can be set by the user, therefore potentially of low order. The controller order depends on the number of observers selected by the user. This is the main difference with traditional robust and conservative controllers that often lead to unnecessary very high order controllers because the control design itself does not consider only the specifications given by the user but many others that are not mastered. This is typically the case with the $H_{\infty}$ control design [31]. In this article, we show a new potential of robustness in AFM with a robust method, where the control constraints are really mastered by a wise choice of the pole assignment and the choice of the worst case models.

2) The gain matrix $K$ obtained from (15) can be structured. Each gain corresponds to an ESA related to a specific LTI model. The designer can master each feedback by adding for instance dynamic filter (e.g., low-pass filter, bandstop filter, and so on) to some feedback if necessary.

In [31], a $H_{\infty}$ controller is designed to control, in scanning mode, the same piezoelectric inertia actuator than those used in this article. The control specifications were almost the same than those specified for the design of the controller 2. In [31], the control specifications were: maximal closed-loop response time lower than $5 \mathrm{~ms}$, vibrations damping with no overshoot, and maximal static error lower than $2 \%$. With the $H_{\infty}$ design, the order of the controller is equal to the order of the open-loop system + the order of weighting functions. In the multimodel design, the order of the controller is equal to the number of the observers +1 . Therefore, the same closed-loop performances have been obtained with a controller of ninth order in [31] with the $H_{\infty}$ approach, while here with the controller 2, a fiveorder controller has been enough. If the $H_{\infty}$ approach is used to design the entire hybrid control structure of Fig. 9, this will lead to a significant computing time. Here, the multimodel design for hybrid control is a real advantage for real-time implementation issues.

\section{COnClusion}

A novel approach for robust hybrid control and its application to atomic force microscopy has been presented in this article. The main advantage of the control strategy is its robustness and its low conservatism. The structure of the hybrid controller has been presented, and each control part has been detailed. The control method has shown its ability to do in an automated and robust way, a complete landing procedure of AFM tip, and a precise characterization of interaction force regions with a bumpless switching under dynamic constraints. The results have shown the robustness of the closed-loop systems against the hysteresis, the uncertainties of the dynamic parameters due to the fabrication process and system aging, and instrumental issues such as the real-time data acquisition. This capability suggests a promising potential in view of using the controller in an industrial context. More generally, the new hybrid control method can be applied to any uncertain and/or nonlinear 
system that can be approximated by a family of linear statespace models and for which switch constraints are needed.

\section{ACKNOWLEDGMENT}

The authors would like to thank Vincent Cailliez for his statistical analysis of the tuning fork signal.

\section{REFERENCES}

[1] G. Binnig, C. F. Quate, and C. Gerber, "Atomic force microscope," Phys. Rev. Lett., vol. 56, pp. 930-933, 1986.

[2] D. Y. Abramovitch, S. B. Andersson, L. Y. Pao, and G. Schitter, "A tutorial on the mechanisms, dynamics, and control of atomic force microscopes," in Proc. Amer. Control Conf., Jul. 2007, pp. 3488-3502.

[3] Y. K. Yong, S. O. R. Moheimani, B. J. Kenton, and K. K. Leang, "Invited review article: High-speed flexure-guided nanopositioning: Mechanical design and control issues," Rev. Sci. Instrum., vol. 83, no. 12, Dec. 2012, Art. no. 121101, doi: 10.1063/1.4765048.

[4] A. J. Fleming, S. S. Aphale, and S. O. R. Moheimani, "A new method for robust damping and tracking control of scanning probe microscope positioning stages," IEEE Trans. Nanotechnol., vol. 9, no. 4, pp. 438-448, Jul. 2010.

[5] M. R. P. Ragazzon, M. G. Ruppert, D. M. Harcombe, A. J. Fleming, and J. T. Gravdahl, "Lyapunov estimator for high-speed demodulation in dynamic mode atomic force microscopy," IEEE Trans. Control Syst. Technol., vol. 26, no. 2, pp. 765-772, Mar. 2018.

[6] S. Belikov, J. Alexander, M. Surtchev, I. Malovichko, and S. Magonov, "Automatic probe landing in atomic force microscopy resonance modes," in Proc. Amer. Control Conf. (ACC), May 2017, pp. 2894-2899.

[7] A. Bolopion, H. Xie, D. S. Haliyo, and S. Regnier, "Haptic teleoperation for 3-D microassembly of spherical objects," IEEE/ASME Trans. Mechatronics, vol. 17, no. 1, pp. 116-127, Feb. 2012.

[8] J.-O. Abrahamians, B. Sauvet, J. Polesel-Maris, R. Braive, and S. Regnier, "A nanorobotic system for in situ stiffness measurements on membranes," IEEE Trans. Robot., vol. 30, no. 1, pp. 119-124, Feb. 2014.

[9] Z. Gong, B. K. Chen, J. Liu, and Y. Sun, "Robotic probing of nanostructures inside scanning electron microscopy," IEEE Trans. Robot., vol. 30, no. 3, pp. 758-765, Jun. 2014.

[10] C. Ru et al., "Automated four-point probe measurement of nanowires inside a scanning electron microscope," IEEE Trans. Nanotechnol., vol. 10 , no. 4, pp. 674-681, Jul. 2011.

[11] U. Mick, V. Eichhorn, T. Wortmann, C. Diederichs, and S. Fatikow, "Combined nanorobotic AFM/SEM system as novel toolbox for automated hybrid analysis and manipulation of nanoscale objects," in Proc. IEEE Int. Conf. Robot. Autom., May 2010, pp. 4088-4093.

[12] A. J. Fleming, "Dual-stage vertical feedback for high-speed scanning probe microscopy," IEEE Trans. Control Syst. Technol., vol. 19, no. 1, pp. 156-165, Jan. 2011

[13] S. Belikov, J. Alexander, M. Surtchev, and S. Magonov, "Digital q-control and automatic probe landing in amplitude modulation phase imaging AFM mode," IFAC-PapersOnLine, vol. 50, no. 1, pp. 10882-10888, Jul. 2017.

[14] M. Boudaoud, T. Lu, S. Liang, R. Oubellil, and S. Regnier, "A voltage/frequency modeling for a multi-DOFs serial nanorobotic system based on piezoelectric inertial actuators," IEEE/ASME Trans. Mechatronics, vol. 23, no. 6, pp. 2814-2824, Dec. 2018.

[15] I. M. Malovichko, "Development and application of an AFM probe soft approach method," Bull. Russian Acad. Sci., Phys., vol. 77, no. 8, pp. 969-971, Aug. 2013.

[16] J. M. Lemos, H. Magalhaes, T. Mendonca, and P. Rocha, "Observer dynamics in switching control: Application to neuromuscular blockade," in Proc. IEEE Int. Workshop Intell. Signal Process., Sep. 2005, pp. 211-216.

[17] G. Qin, Z. Duan, and G. Chen, "LQ bumpless transfer between two tracking controllers," Int. J. Control, vol. 85, no. 10, pp. 1546-1556, Oct. 2012

[18] J. J. Yamé and M. Kinnaert, "On bumps and reduction of switching transients in multicontroller systems," Math. Problems Eng., vol. 2007, pp. 1-17, 2007.

[19] M. Pasamontes, J. D. Álvarez, J. L. Guzmán, J. M. Lemos, and M. Berenguel, "A switching control strategy applied to a solar collector field," Control Eng. Pract., vol. 19, no. 2, pp. 135-145, Feb. 2011.

[20] Y. Peng, D. Vrancic, and R. Hanus, "Anti-windup, bumpless, and conditioned transfer techniques for PID controllers," IEEE Control Syst. Mag., vol. 16, no. 4, pp. 48-57, Aug. 1996.
[21] J. M. A. Lourenco and J. M. Lemos, "Learning in multiple model adaptive control switch," IEEE Instrum. Meas. Mag., vol. 9, no. 3, pp. 24-29, Jun. 2006.

[22] M. Kinnaert, T. Delwiche, and J. Yame, "State resetting for bumpless switching in supervisory control," in Proc. Eur. Control Conf. (ECC), Aug. 2009, pp. 2097-2102.

[23] J. Steenis and A. Dumais, "Bumpless control for reduced THD in power factor correction circuits," in Proc. IEEE Appl. Power Electron. Conf. Expo. (APEC), Mar. 2016, pp. 3219-3223.

[24] Y. Qi and W. Bao, "Robust bumpless transfer design using adaptive sliding mode approach," Asian J. Control, vol. 15, no. 6, pp. 1785-1793, Nov. 2013.

[25] C. A. Lopez Martinez, R. van de Molengraft, and M. Steinbuch, "High performance teleoperation by bumpless transfer of robust controllers," in Proc. IEEE Haptics Symp. (HAPTICS), Feb. 2014, pp. 209-214.

[26] C. Döll, Y. Le Gorrec, G. Ferreres, and J. F. Magni, "A robust self-scheduled missile autopilot: Design by multi-model eigenstructure assignment," Control Eng. Pract., vol. 9, no. 10, pp. 1067-1078, Oct. 2001

[27] Y. Le Gorrec and C. Chiappa, "Controller parametric robustification using observer-based formulation and multimodel design technique," IEEE Trans. Autom. Control, vol. 50, no. 4, pp. 526-531, Apr. 2005.

[28] M. Boudaoud, Y. Le Gorrec, Y. Haddab, and P. Lutz, "Gain scheduling control of a nonlinear electrostatic microgripper: Design by an eigenstructure assignment with an observer-based structure," IEEE Trans. Control Syst. Technol., vol. 23, no. 4, pp. 1255-1267, Jul. 2015.

[29] M. Boudaoud et al., "Robust microscale grasping through a multimodel design: Synthesis and real time implementation," Control Eng. Pract., vol. 39, pp. 12-22, Jun. 2015.

[30] T. Akiyama, U. Staufer, N. F. de Rooij, P. Frederix, and A. Engel, "Symmetrically arranged quartz tuning fork with soft cantilever for intermittent contact mode atomic force microscopy," Rev. Sci. Instrum. vol. 74, no. 1, pp. 112-117, Jan. 2003, doi: 10.1063/1.1523631

[31] R. Oubellil, A. Voda, M. Boudaoud, and S. Régnier, "Mixed stepping/scanning mode control of stick-slip SEM-integrated nano-robotic systems," Sens. Actuators A, Phys., vol. 285, pp. 258-268, Jan. 2019.

[32] J. Cailliez, M. Boudaoud, S. Liang, and S. Regnier, "A multi-model design for robust hybrid control of non-linear piezoelectric actuators at the micro/nano scales," in Proc. Annu. Amer. Control Conf. (ACC), Jun. 2018, pp. 5131-5137.

[33] B. C. Moore and G. Klein, "Eigenvector selection in the linear regulator problem: Combining modal and optimal control," in Proc. IEEE Conf. Decis. Control Including 15th Symp. Adapt. Processes, Dec. 1976, pp. 214-215.

[34] J.-F. Magni, "An observer based multimodel control design approach," Int. J. Syst. Sci., vol. 30, no. 1, pp. 61-68, Jan. 1999.

[35] K. J. Åström and T. Hägglund, Advanced PID Control, vol. 461. ISA-The Instrumentation, Systems, and Automation Society Research Triangle, 2006. [Online]. Available: https://www.isa.org/store/advancedpid-control/116187

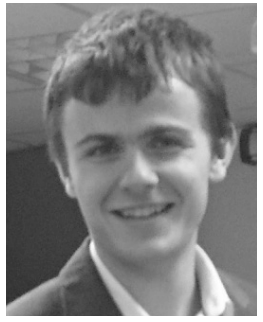

Jonathan Cailliez received the master's degree in electrical engineering from the ENS Cachan, Cachan, France, and the master's degree in industrial informatics from Université Pierre et Marie Curie (UPMC), Paris, France, in 2017, where he is currently pursuing the $\mathrm{Ph} . \mathrm{D}$. degree with the Institut des Systèmes Intelligents et de Robotique (ISIR).

His current research interests include microrobotics and robust control of nonlinear and/or uncertain systems. 


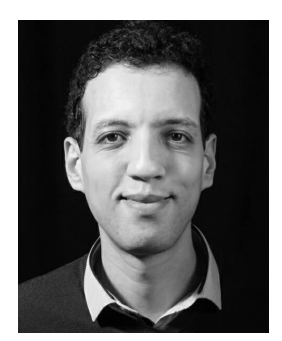

Mokrane Boudaoud received the Engineering degree in automatic control from the University of Science and Technology Houari Boumédiène (USTHB), Algiers, Algeria, in 2007, the M.S. degree in mechatronics and microrobotics from the University of Franche-Comté (UFC), Besançon, France, in 2009, and the Ph.D. degree in control engineering from the Femto-St Institute, Besançon, France, in 2012.

He is currently an Associate Professor (Maître de Conférences) with Sorbonne University, Paris, France, with research affiliation at the Institut des Systèmes Intelligents et de Robotique (ISIR). His main fields of interest are micro/nanorobotics, robust control, and nonlinear modeling.

Dr. Boudaoud has been an Associate Editor of the journal Mechatronics (Elsevier) since 2017

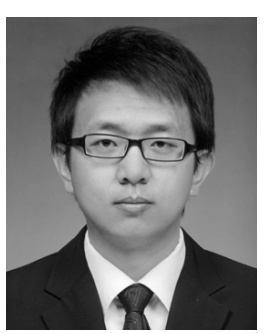

Shuai Liang was born in Changchun, China, in 1989. He received the bachelor's degree in mechanical engineering from the Changchun University of Science and Technology, Changchun, in 2012, and the master's degrees in mechanical engineering from the Harbin Institute of Technology (HIT), Harbin, China, in 2014. He is currently pursuing the Ph.D. degree with the Institute for Intelligent Systems and Robotics (ISIR), University Pierre and Marie Curie (UPMC), Paris, France.

His current research interests include micromanipulation robotics, microscanning, and micropositioning.

Mr. Liang won the Best Paper Finalist in the International Conference of Mechanical and Automation (ICMA) 2014.

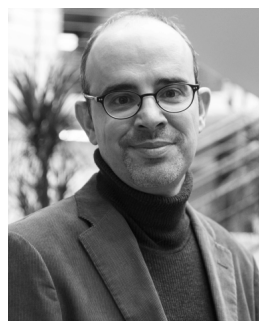

Stéphane Régnier received the $\mathrm{Ph} . \mathrm{D}$. degree in mechanics and robotics from the University of Pierre and Marie Curie, Paris, France in 1996.

$\mathrm{He}$ is currently a Professor with the Institute of Intelligent Systems and Robotics (ISIR), Sorbonne University, Paris. He has been the Head of the ISIR Micromanipulation Team since 2001. His research interests are focused on micro and nano manipulation, teleoperation, and haptic feedback at the nanoscale, micromechatronics, and biological cell characterization. 